CER-ETH - Center of Economic Research at ETH Zurich

\author{
Life-Cycle Preferences Revisited
}

A. Bommier

Working Paper 11/155

December 2011

Economics Working Paper Series

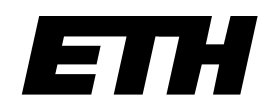

Eidgenössische Technische Hochschule Zürich Swiss Federal Institute of Technology Zurich 


\title{
Life-Cycle Preferences Revisited *†
}

\author{
Antoine Bommier \\ ETH-Zurich
}

December 21, 2011

\begin{abstract}
An axiomatic construction for lifecycle preferences accounting for the finiteness and the randomness of life duration is provided. We emphasize the role of intertemporal correlation aversion and explain why multiplicative preferences provide an interesting alternative to additive preferences, allowing to separate risk aversion from intertemporal elasticty of substitution. Implications regarding the shape of the optimal consumption profiles are discussed.
\end{abstract}

Keywords : Intertemporal choice, Life-cycle models, Multivariate risk aversion, Uncertain lifetime.

JEL classification : D91

*I am grateful to Didier Blanchet, Martin Browning, Michele Cohen, Pierre Dubois, Thibault Gajdos, Roger Guesnerie, Dirk Krueger, Ronald Lee, Thierry Magnac, Humberto Moreira, Pierre Pestieau, Jean-Charles Rochet, Guy Stecklov, Jean-Marc Tallon, Jean-Christophe Vergnaud, Bertrand Villeneuve and Peter Wakker for helpful discussions and suggestions. I have also benefited from the participants of the 58th Econometric Society European Meeting, the Second Workshop of the RTN Project on Economics of Ageing in Europe, the séminaire d'Economie Théorique of the University of Toulouse and the Journées Jourdan. Previous versions of this paper - using continuous time modeling - circulated under the titles "Valuing Life under the Shadow of Death" and "Life-Cylcle Theory for Human Beings". Jesús FernándezVillaverde and Dirk Krueger kindly sent me the data which was used for plotting Figures 2 and 3. Comments and suggestions from one associate editor and five anonymous referees were most helpful to get this last version. Financial support from Swiss Re is gratefully acknowledged.

$\dagger$ All correspondence should be sent to Antoine Bommier, Chair of Integrative Risk Management and Economics at ETH-Zurich, Zurichbergstrasse 18, 8032 Zurich, Switzerland. Email: abommier@ethz.ch. 


\section{Introduction}

Looking at the economic literature one may distinguish two main categories of theoretical works on intertemporal preferences: those that consider an infinite horizon and those that consider a finite horizon. In between the two, however, lies an intermediate setting, which has barely been studied to date: the case of an infinite possibility of finite horizons. This corresponds to the case of agents who care for flows of payments, which will stop after some finite time, but whose stopping time is random or a matter of choice.

The most obvious case where such a setting is relevant is life cycle planning. Human beings are indeed sure to die within a finite time, but do not know when they will die. They have therefore to consider an infinite possibility of finite horizons. A number of other problems, however, such as firm behavior, dynasty behavior, political modeling, could also fit into this domain.

The economic literature has not paid special attention to this setting because it is perceived as a particular instance of the infinite horizon case. The standard argument is that a finite payment flow can always be seen as an infinite payment flow where payments equal zero after some finite time. Thus, assuming an infinite horizon would provide a general framework allowing to account for cases where payments have to be null after some finite amount of time. However, the economic literature, in particular that on social choice, abounds with examples where it is found that working on too large a domain may well lead to too restrictive results. The present paper provides an illustration of this general principle in the domain of intertemporal choice. We will indeed emphasize that considering the set of finite payment flows, rather than the larger set of infinite payment flows, makes it possible to derive new preference representations, with potentially important implications.

Our contribution is threefold. First, we will suggest an axiomatic construction of preferences based on very simple axioms: rationality, continuity, independence (in the sense introduced by von Neumann and Morgenstern) and stationarity. This will provide the foundations for widely used models (as with Yaari's model of intertemporal choice under uncertain lifetime) as well as suggest new speci- 
fications for life-cycle preferences. In particular, we will underline the case of multiplicative preferences which provide a way to disentangle intertemporal elasticity of substitution and risk aversion, without abandoning the expected utility framework or the assumption of preference stationarity.

Second we discuss fundamental properties of stationary life-cycle preferences and discuss how they relate to what can be observed from choices of agents having an uncertain lifetime. We will in particular provide a formal decomposition of the rate of time discounting, explaining how pure time preferences, mortality and risk aversion contribute to human impatience.

Last, as an illustration we will apply our framework to life cycle consumption planning. The standard result, obtained when considering additive preferences and perfect credit markets, is that people with stationary preferences should opt for a consumption profile which has a constant growth rate (see Yaari, 1965). However, this prediction was contradicted by empirical observations, where it is found that the life cycle consumption profile is hump shaped. Allowing for temporal risk aversion, we find that Yaari's predictions do not extend to nonadditive preferences. The increase of mortality rates at old age together with a positive intertemporal correlation aversion may generate non-monotonic consumption profiles - even when focusing on stationary preferences and considering perfect markets.

The paper is organized as follow. Section 2 discusses the related literature. Section 3 details the setting, making clear what is meant by an infinite possibility of finite horizons. Sections 4 and 5 provide an axiomatic construction of preferences. Properties of stationary preferences are then discussed in Sections 6 and 7. Section 8 is about time discounting when agents' horizons are random. Section 9 looks at the problem of consumption smoothing when considering realistic mortality data. Section 10 concludes.

\section{Related Literature}

The axiomatic construction of preferences developed in the current paper relies on an assumption of stationarity of preferences. Such an assumption was first 
introduced by Koopmans (1960) in a deterministic setting. It was extended by Epstein (1983) to the case of choice under uncertainty within the expected utility framework, and by Epstein and Zin (1989) to non-expected utility models. As our work fits into the expected utility framework, our formulation of preference stationarity will be exactly the same as the one proposed by Epstein (1983). We will also make use of the same recursivity arguments to provide an explicit representation of preferences. The originality lies therefore neither in the stationarity assumption nor in the techniques used, but on the fact that we consider a domain that is smaller than usual. As we will see, this explains why even if our results look similar to those of Epstein (1983) in some respects, they also differ in suggesting models that could not be obtained when considering infinite horizons. Even if the idea of constraining consumption to be in a given state after a finite amount of time has been used in several instances (starting from Ramsey, 1928, could we say ${ }^{1}$ ), we are not aware of other papers providing an axiomatic construction of preferences on the domain we consider.

One of the findings of the paper is that stationary preferences can have a multiplicative representation, making it possible to study the role of risk aversion, keeping intertemporal substitutability constant. As a consequence, our work relates to the literature devoted to studying the role of risk aversion in intertemporal frameworks, and in particular to Kihlstrom and Mirman (1974) and Epstein and Zin (1989). The relevance of these different approaches is now discussed in Bommier, Chassagnon, Le Grand (2010), who propose an abstract definition of what it means "to be more risk averse than someone else" and examines which classes of utility functions are well ordered in terms of risk aversion.

Since the preferences we derive are not necessarily time additive, we will often refer to the literature on multi-attribute utility theory. For example, the notion of "intertemporal correlation aversion" (ICA, hereafter), which is explained in Section 6, was discussed -under different names- in Keeney and Raiffa (1976), Richard (1975), Epstein and Tanny (1980), Dorfleitener and Krapp (2007) or Tsetlin and

\footnotetext{
${ }^{1}$ Ramsey's assumption is slighty different as he considers the case where utility has to reach a given level -called Bliss- in a finite amout of time or "at least approximate to it indefinitely" (Ramsey, 1928 p.545).
} 
Winkler (2009). Bommier (2007) showed how ICA, risk aversion and intertemporal elasticity of substitution are related. Eeckhoudt, Rey and Schlessinger (2007) explained how ICA could be identified through simple experiments. Recently, Andersen, Harrison, Lau and Ruström (2011) provide experimental results measuring ICA.

Allowing for ICA generates a significant shift in life cycle modeling, in particular when accounting for lifetime uncertainty. Bommier (2006) first highlighted that the combination of ICA (or risk aversion with respect to life duration) and mortality risk generates time discounting, in a paper which focused on weakly separable preferences. Formal results on the rate of time discounting were derived, but only in the limit case where the value of life tends towards infinity, so that additivity of the expected utility function could be recovered. One of the results of the present paper also bears on time discounting under lifetime uncertainty. Departing from the focus of Bommier (2006), it applies to the whole set of stationary preferences (including those which are not weakly separable) and is independent of any asymptotic approximation. We will indeed provide a general decomposition of the rate of time discounting into elements that arise from pure time preference, mortality and risk aversion.

As ICA generates a strong link between mortality risks and impatience, opting for models with non-zero ICA provides new insights on a number of topics, such as the impact of mortality decline, the value of life or lifecycle portfolio choice. These points are discussed in other papers: Bommier (2008), for the impact of mortality decline, Bommier and Villeneuve (2010) for the value of life, Bommier and Rochet (2006) and Bommier (2010) for issues related to portfolio choice. ${ }^{2}$ Giving up additive separability may also lead to new policy recommendations when there is asymmetric information. Such a point is addressed in Bommier, Leroux and Lozachmeur (2010), who consider the design of pension systems when agents have private information on their mortality.

There are actually many other applications that could be developed to illustrate the role of ICA. We shall only mention one of them in the current paper:

\footnotetext{
${ }^{2}$ Bommier and Rochet (2006) ignore lifetime uncertainty. Bommier (2010) introduces random mortality, but focuses on weakly separable preferences.
} 
the possibility of providing an explanation for the hump shape of life cycle consumption profiles. In that perspective, the paper relates to the empirical literature that estimates the shape of consumption profiles, as with Gourinchas and Parker (2002) or Fernández-Villaverde and Krueger (2007). It also connects with those who suggested explanations for this hump shape (see for example Attanasio, Banks, Meghir and Weber, 1999), and more particularly those who wondered whether mortality could be at the origin of this shape, such as Bütler (2001), Hansen and Imrohoroglu (2008) or Feigenbaum (2008).

To end this Section we shall emphasize that this paper fits into the literature on intertemporal choice under uncertainty, which has developed over several decades, providing numerous contributions that cannot be extensively reviewed here. The way our paper innovates in this domain is rather atypical, in the sense that we do not extend an existing framework. Many previous developments involved looking at what happens when relaxing some assumption. The literature on hyperbolic discounting on habit formation, or on rank dependent expected utility, are examples illustrating what can be achieved by relaxing some "classical" assumptions. Rather than following the same line, the current paper focuses on strong and standard assumptions, but innovates by defining preferences on a smaller domain. This is definitely not to mean that these classical assumptions should not be weakened in future works. On the contrary, it would be extremely interesting to consider non-stationary preferences or non-expected utility approaches. But, instead of focusing on extensions of the additive model, as has been done so far, one could also build upon the models that are suggested in the paper, and in particular upon the multiplicative model. In other words, while emphasizing that there are some interesting specifications which were not developed, though compatible with the most standard assumptions, the paper does not want to argue that departures from the "classical" assumptions should be disregarded. Instead, it aims at providing a different basis, upon which extensions could be developed. 


\section{Setting}

Time is assumed to be discrete. We are interested in the problems of agents who care for a flow of consumption (or payments) but who know that, for some reason, this flow will have to stop after a finite amount of time. Speaking of life and death is probably the easiest way to illustrate our setting, but it is clear that our work does not exclusively apply to the modelling of human beings. It could also apply to firms, dynasties, political parties or other sort of agents.

An agent will be considered to be "alive" when consuming a positive amount and "dead" when the consumption flow has come to its end.

Formally, consumption is assumed to take values in $\mathbb{R}_{+}^{*}$ and there is a death state denoted by the letter $d$. Thus, at any moment in time, the state of an individual is an element of the set:

$$
X=\mathbb{R}_{+}^{*} \cup\{d\}
$$

Technically speaking, we could confuse being dead and having zero consumption. This could however be misleading. We would indeed end up working with a connected, convex and ordered set $X$ and tempted to impose standard assumptions of continuity, convexity or monotonicity of preferences. However, in many cases these assumptions could not relate to any sensible or plausible statement. Think of human life for example: it makes no sense to think of a state where the agent is half dead and half alive. A lottery that gives one unit of consumption $(x=1)$ or being dead $(x=d)$ with equal probabilities does not need to have a certainty equivalent. This seems clear when considering that death is not like any consumption level, while it could look counter-intuitive if we had artificially set $d=0$. Similarly, with our approach, there may exist a consumption level $c^{*}>0$ providing the same utility as death. The existence of such a consumption level, and its value, are determined by the agent's preferences and not imposed by construction. Had we artificially set $d=0$, we would find that an agent could be indifferent between consuming $c^{*}>0$ and "consuming" 0 , which could seem odd, if one forgets that "zero consumption" actually means being dead - something 
different from being alive and consuming nothing.

Considering $\left(z_{i}\right)_{i \in \mathbb{N}} \in X^{\mathbb{N}}$ an infinite sequence of elements of $X$, we shall say that $\left(z_{i}\right)_{i \in \mathbb{N}}$ is a "life" (or a "feasible flow of payments") whenever it fulfills the two following properties:

Property 1: (Life finiteness) There exist $j \in \mathbb{N}$ such that $z_{j}=d$.

Property 2: (Death Irreversibility) If $z_{j}=d$ for some $j \in \mathbb{N}$, then $z_{k}=d$ for all $k \geq j$.

We will denote by $Z$ the set of lives, that is the set of elements of $X^{\mathbb{N}}$ which fulfill the above two properties. Note that with this definition the sequence $(d, d, \ldots)$ is considered as a life. An important feature of this set $Z$ is that it is stationary in the sense that

$$
\left(z_{0}, z_{1}, z_{2}, \ldots\right) \in Z \Longrightarrow\left(z_{1}, z_{2}, \ldots\right) \in Z
$$

This property is essential as it will enable the notion of preference stationarity to be defined (Axiom 4, introduced later on).

We denote by $L(Z)$ the set of simple lotteries (i.e: lotteries with a finite number of possible outcomes) with outcomes in $Z$. This set of lotteries is endowed with a mixture operation $\oplus$, defined as usual.

Moreover, for any $l \in L(Z)$ and any $c_{0}$ in $\mathbb{R}_{+}^{*}$ we define

$$
c_{0} * l \in Z
$$

as the lottery that involves consuming $c_{0}$ for sure in the first period and then living (and dying) according to the lottery $l$, delayed by one period. For example, if $l$ is the lottery that gives $\left(x_{0}, x_{1}, x_{2} \ldots\right)$ or $\left(y_{0}, y_{1}, y_{2} \ldots\right)$ with equal probability, then $c_{0} * l$ is the lottery that gives $\left(c_{0}, x_{0}, x_{1}, x_{2} \ldots\right)$ and $\left(c_{0}, y_{0}, y_{1}, y_{2} \ldots\right)$ with equal probability.

\section{Assumptions}

We now state the axioms that will lead to our representation result. 
Axiom 1 (Ordering) Individuals have a rational preference relation (i.e. a complete preorder) on $L(Z)$.

We denote by $\succeq$ the preference relation, $\succ$ the strict preference relation, and $\sim$ the indifference relation.

Axiom 2 (Independence) For any $l_{1}, l_{2}, l_{3} \in L(Z)$ and any $\lambda \in(0,1)$

$$
l_{1} \succeq l_{2} \Leftrightarrow \lambda l_{1} \oplus(1-\lambda) l_{3} \succeq \lambda l_{2} \oplus(1-\lambda) l_{3}
$$

Axiom 3 (Continuity) For any $l_{1}, l_{2}, l_{3} \in L(Z)$ such that

$$
l_{1} \succ l_{2} \succ l_{3}
$$

there exists $\lambda \in(0,1)$ such that

$$
l_{2} \sim \lambda l_{1} \oplus(1-\lambda) l_{3}
$$

Axioms 1 to 3 are the usual axioms of expected utility theory. As such, they could be open to of discussion. A number of empirical and experimental papers have stressed that the independence axiom is probably too strong. Several theoretical frameworks have been suggested relaxing the independence axiom, as with rank dependent expected utility theory. It would of course be interesting to develop extensions of the current paper applying these approaches, but that is a matter for the future.

The last axiom expresses the assumption of stationarity.

Axiom 4 (Stationarity) For any $c_{0} \in \mathbb{R}_{+}^{*}$ and any $l, l^{\prime} \in L(Z)$ we have:

$$
l \succeq l^{\prime} \Leftrightarrow\left(c_{0} * l\right) \succeq\left(c_{0} * l^{\prime}\right)
$$

In a dynamic setting, the assumption of stationarity would imply that preferences are history independent and time consistent. Therefore, two individuals of different ages, say a 30 year old and 60 year old, would have the same preferences regarding their futures. That does not imply that they would behave in 
the same way. They would do so only if they faced the same constraints: that is the same budget constraints and the same mortality risks. In practice mortality strongly depends on age, and a 30 year old and a 60 year old are confronted with radically different constraints. Consequently, we expect them to behave very differently, even if they have the same preferences. Stated otherwise, stationarity involves assuming that age is a relevant variable only because it affects individual constraints (in particular those related to mortality). Such an assumption can of course be criticized. The literature on habit formation emphasizes that past consumption may impact preferences regarding the future. Departing from the stationarity assumption to introduce history dependence would surely be another interesting extension to develop. The present paper, which focus on stationary preferences, should thus be seen as a starting point providing economic insights on how individual behavior may change along the life cycle as a direct consequence of decreasing life expectancy, independent of any changes in taste.

An important feature of our axiomatic formulation is that it applies to the set of atemporal lotteries and uses the axioms introduced by von Neumann and Morgenstern. Therefore, we remain within the realm of expected utility theory and do not follow the direction initiated by Kreps and Porteus (1978) who extended the von Neumann-Morgenstern framework to temporal lotteries in order to obtain more general models of dynamic choice. Corollary 3 of Kreps and Porteus (1978) tells us that our framework can be considered as a particular case of Kreps and Porteus's dynamic choice theory where individuals are indifferent to the timing of resolution of uncertainty. Whether or not such an assumption of indifference should be relaxed is open to debate. Dynamic choice theory indisputably offers a greater flexibility. But it is also much more complex than expected utility theory. In fact, most papers that use dynamic choice theory respond to this increase in complexity by assuming particular specifications. Instead, we prefer to remain in the simpler framework provided by the expected utility theory but consider all the specifications that are consistent with the stationarity axiom. 


\section{The set of stationary preferences}

As is known from Fishburn (1970), Axioms 1 to 3 imply that preferences on $L(Z)$ have an expected utility representation, which means that there exists a function

$$
U: Z \rightarrow \mathbb{R}
$$

such that

$$
l_{1} \succeq l_{2} \Leftrightarrow E_{l_{1}}[U] \geq E_{l_{2}}[U]
$$

where $E_{l_{1}}$ [.] and $E_{l_{1}}$ [.] denote the expectation operator associated with the lotteries $l_{1}$ and $l_{2}$. In the following we will call $U$ a utility index. Our first result provides the structure that this utility index must have for preferences to be stationary :

Proposition 1 Preferences fulfill Axioms 1 to 4 if and only if they can be represented in the expected utility framework with a utility index $U$ such that

$$
U(z)=\sum_{i=0}^{+\infty} u\left(z_{i}\right) \exp \left(-\sum_{j=0}^{i-1} v\left(z_{j}\right)\right) \text { for all } z=\left(z_{i}\right)_{i \in \mathbb{N}} \in Z
$$

where $u$ and $v$ are two functions from $X$ into $\mathbb{R}$, with $u(d)=0 .^{3}$

\section{Proof. 1. Sufficiency}

Consider two functions $u$ and $v$ from $X$ into $\mathbb{R}$ with $u(d)=0$ and the utility index $U(z)=\sum_{i=0}^{+\infty} u\left(z_{i}\right) \exp \left(-\sum_{j=0}^{i-1} v\left(z_{i}\right)\right)$. For any $c_{0} \in \mathbb{R}_{+}$, and any $l \in L(Z)$ we have:

$$
E_{c_{0} * l}[U]=u\left(c_{0}\right)+\exp \left(-v\left(c_{0}\right)\right) E_{l}[U]
$$

and therefore, for any $l, l^{\prime}$ and $c_{0}$

$$
l \succeq l^{\prime} \Leftrightarrow E_{l}[U] \geq E_{l^{\prime}}[U] \Leftrightarrow E_{c_{0 * l}}[U] \geq E_{c_{0} * l^{\prime}}[U] \Leftrightarrow c_{0} * l \succeq c_{0} * l^{\prime}
$$

\footnotetext{
${ }^{3}$ When $i=0$, the second sum in equation (1) reads as $\sum_{j=0}^{-1}$, which, by convention, should be considered as being equal to zero. Such a convention will be used throughout the paper. Similarly, for products, we will use the convention $\prod_{j=0}^{-1}=1$.
} 
Preferences are thus stationary.

\section{Necessity}

Axioms 1 to 3 imply that preferences admit an expected utility representation (Fishburn 1970). Let us denote by $U$ a utility index associated with such a representation. As adding a constant to $U$ does not impact preferences, it can be assumed without loss of generality that $U(d, d, \ldots)=0$. Let us now show that $U$ may be expressed as in (1) for some functions $u$ and $v$.

For any $c_{0} \in \mathbb{R}_{+}$and any $l, l^{\prime} \in L(Z)$, we know from Axiom 4 that:

$$
l \succeq l^{\prime} \Leftrightarrow\left(c_{0} * l\right) \succeq\left(c_{0} * l^{\prime}\right)
$$

With the expected utility representation:

$$
\left(c_{0} * l\right) \succeq\left(c_{0} * l^{\prime}\right) \Leftrightarrow E_{c_{0} * l}[U] \geq E_{c_{0} * l^{\prime}}[U]
$$

For a given $c_{0}$ define:

$$
\begin{aligned}
V & : \quad Z \rightarrow \mathbb{R} \\
z & =\left(z_{i}\right)_{i \in \mathbb{N}} \Rightarrow V(z)=U\left(c_{0}, z_{0}, z_{1}, \ldots\right)
\end{aligned}
$$

For any $l \in L(Z)$ we have $E_{c_{0} * l}[U]=E_{l}[V]$. Thus:

$$
l \succeq l^{\prime} \Leftrightarrow E_{l}[V] \geq E_{l^{\prime}}[V]
$$

which means that $V$ is another utility index representing the preference relation.

Since, in the expected utility representation, two utility indices represent the same preferences if and only if they are related by a positive affine transformation, there must exist $u\left(c_{0}\right) \in \mathbb{R}$ and $\omega\left(c_{0}\right)>0$, such that, for all $\left(z_{i}\right)_{i \in \mathbb{N}} \in Z$ :

$$
V\left(z_{0}, z_{1}, \ldots\right)=u\left(c_{0}\right)+\omega\left(c_{0}\right) U\left(z_{0}, z_{1}, \ldots\right)
$$


By definition of the function $V$, we thus have:

$$
U\left(c_{0}, z_{0}, z_{1}, \ldots\right)=u\left(c_{0}\right)+\omega\left(c_{0}\right) U\left(z_{0}, z_{1}, \ldots\right)
$$

Since $\omega\left(c_{0}\right)>0$ we can define $v\left(c_{0}\right)=-\log \left(\omega\left(c_{0}\right)\right)$, to obtain:

$$
U\left(c_{0}, z_{0}, z_{1}, \ldots\right)=u\left(c_{0}\right)+\exp \left(-v\left(c_{0}\right)\right) U\left(z_{0}, z_{1}, \ldots\right)
$$

Now, because of life finiteness and death irreversibility, any element of $Z$ different from $(d, d, \ldots)$ is of the form $\left(c_{0}, c_{1}, \ldots, c_{n}, d, d, d, \ldots\right)$ for some $n \in \mathbb{N}$ and some $c_{i} \in \mathbb{R}_{+}^{*}$. Iterating (2) $n$ times, we obtain:

$$
\begin{aligned}
U\left(c_{0}, c_{1}, \ldots, c_{n}, d, d, d, \ldots\right)= & u\left(c_{0}\right)+\sum_{j=1}^{n} u\left(c_{j}\right) \exp \left(-\sum_{k=1}^{j-1} v\left(c_{k}\right)\right) \\
& +\exp \left(-\sum_{k=1}^{n} v\left(c_{k}\right)\right) U(d, d, d, \ldots) \\
= & \sum_{j=1}^{n} u\left(c_{j}\right) \exp \left(-\sum_{k=1}^{j-1} v\left(c_{k}\right)\right)
\end{aligned}
$$

Extending the function $u$, which was defined on $\mathbb{R}_{+}^{*}$, to $X$ by posing $u(d)=0$ we obtain that for all $\left(z_{i}\right)_{i \in \mathbb{N}} \in Z$

$$
U(z)=\sum_{i=0}^{+\infty} u\left(z_{i}\right) \exp \left(-\sum_{j=0}^{i-1} v\left(z_{i}\right)\right)
$$

which proves the Proposition.

Before discussing this representation result and explaining how it relates to past contributions, let us give formal names to two particular cases that will be of special importance throughout the paper.

Definition 1 Preferences will be called "additive" if they can be represented with a utility index of the form (1) with a constant function $v$. In such a case, setting 
$\beta=e^{-v}$, equation (1) can be rewritten:

$$
U(z)=\sum_{i=0}^{+\infty} \beta^{i} u\left(z_{i}\right)
$$

Definition 2 Preferences will be said to be "multiplicative" if they can be represented with a utility index of the form (1) with functions $u$ and $v$ such that $v=$ $-k w(c)$ and $u(c)=\frac{1-e^{-k w(c)}}{k}$ for some constant $k$ and some function $w: X \rightarrow \mathbb{R}$ with $w(d)=0$. In such a case, equation (1) can be rewritten:

$$
\begin{aligned}
& U(z)=\frac{1}{k}\left(1-\exp \left(-k \sum_{i=0}^{+\infty} w\left(z_{i}\right)\right)\right) \text { if } k \neq 0 \\
& U(z)=\sum_{i=0}^{+\infty} w\left(z_{i}\right) \text { if } k=0
\end{aligned}
$$

Now, let us discuss the interest of Proposition 1 and the novelty of our approach. A representation result that looks very similar was provided by Epstein (1983). The similarity between Epstein's representation and ours is not surprising since both Epstein (1983) and the current paper deal with stationary expected utility preferences. The assumption of preference stationarity imposes a recursive structure to the utility index, as shown in equation (2), which leads to the general expression given in Proposition $1 .^{4}$ The difference with the representation of Epstein (1983), however, is that we do not have constraints on the function $u$ and $v$, while in Epstein (1983) it is found that $v$ must be positive and $u$ and $\left(1-e^{-v}\right)$ should not be proportional. This is because Epstein deals with infinitely long lived agents, which makes it impossible to obtain well-behaved representations where agents have no time preferences - as is known from Diamond (1965).

The constraints on $u$ and $v$ in Epstein (1983) lead to ruling out the multiplicative model, which is precisely obtained whenever $v=0$ or when $u$ and $\left(1-e^{-v}\right)$ are proportional. However, the multiplicative specification becomes possible in our framework, which accounts for the finiteness of human life. Multiplicative representations of preferences are not new to the economic literature. They were advocated by several theoretical papers (in particular by Richard, 1975) and used

\footnotetext{
${ }^{4}$ The first appearance of this recursive structure may be attributed to Uzawa (1968) who suggested a similar specification in continuous time.
} 
to deal with very concrete issues (Pye, 1973, Ahn, 1989, van der Ploeg, 1993). But these multiplicative representations remained unpopular. When they were used in an infinite horizon setting, they were augmented with an exogenous time discounting factor, and then explicitly disregarded for being non-stationary (see for example the discussion in Epstein and Zin, 1989, p. 951).

Our results emphasize that multiplicative preferences can be stationary. Moreover, they emerge from the set of stationary preferences as being of particular interest. First, as we will see, the multiplicative model appears to be one of the two polar cases that combine stationarity and separability, the other being the usual additive specification. Secondly, the multiplicative model provides a straightforward way to separate intertemporal substitution and risk aversion within the stationary and expected utility framework. By increasing the constant $k$ in the equation (4) that defines the multiplicative utility function, one increases risk aversion without changing ordinal preferences. The multiplicative model therefore makes it possible to analyze the role of risk aversion in intertemporal problems, without abandoning the expected utility framework or the stationarity assumption.

\section{Properties of stationary preferences}

The general form of the von Neumann-Morgenstern utility functions that represent stationary preferences is given in equation (1). However, the meanings of the functions $u$ and $v$ that appear in this formulation are not obvious. For example, we know that when $v$ is constant it represents the rate of time preference. But it is not clear what the rate of time preference is when $v$ is not constant. Similarly, it is not trivial, a priori, to interpret the derivative of $v$, etc. This section provides general definitions of two important concepts -time preference and ICAand deduces what their expressions turn out to be when the utility function has the form given in (1). The meanings of $u$ and $v$ will then become clearer. The additive and multiplicative specifications will then appear as corresponding to fundamental assumptions.

In order to avoid technical difficulties we make two additional assumptions. 
Assumption 1 The functions $u$ and $v$ that appear in equation (1) are twice continuously differentiable.

Assumption 2 The functions $u$ and $v$ are such that for any life $\left(z_{i}\right)_{i \in \mathbb{N}}$ and any age $t$ such that $z_{t} \neq d$ we have:

$$
\frac{\partial U(z)}{\partial z_{t}} \neq 0
$$

Time preference is a familiar notion, usually measured by the rate of time preference. The rate of time preference at time $t$ is usually defined from the marginal rate of substitution between consumption at two subsequent periods of time, when controlling for variation in consumption. More precisely if we take a life $\left(z_{i}\right)_{i \in \mathbb{N}}$ and time $t$ such that $z_{t} \neq d$ we define the rate of time preference at time $t$ by:

$$
R T P_{t}(z)=1-\left.\frac{\frac{\partial}{\partial z_{t+1}} U(z)}{\frac{\partial}{\partial z_{t}} U(z)}\right|_{z_{t+1}=z_{t}} .
$$

The rate of time preference simply describes how marginal utility of consumption varies along the life cycle when controlling for variations in consumption. With the utility index provided in (1) simple derivations lead to:

$$
\operatorname{RTP}_{t}(z)=\frac{u^{\prime}\left(z_{t}\right)\left(1-e^{-v\left(z_{t}\right)}\right)-v^{\prime}\left(z_{t}\right) u\left(z_{t}\right) e^{-v\left(z_{t}\right)}}{u^{\prime}\left(z_{t}\right)-v^{\prime}\left(z_{t}\right) \sum_{i=t+1}^{+\infty} u\left(z_{i}\right) \exp \left(-\sum_{j=t}^{i-1} v\left(z_{j}\right)\right)}
$$

With the additive specification, $R T P_{t}(z)=1-\beta$, which explains the usual interpretation of $\beta$. The multiplicative model provides $R T P_{t}(z)=0$, consistent with the fact that ordinal preferences, independent of $k$, can be represented by $\sum_{i=0}^{+\infty} w\left(z_{i}\right)$

The notion of intertemporal correlation aversion (ICA) is much less well-known, although it has occasionally featured in the economic literature, as mentioned in Section 2. We use the three words "intertemporal correlation aversion" to stress that it corresponds to a particular measure of correlation aversion that can be defined in the intertemporal framework.

Correlation aversion, which itself is not very well known, is a natural concept when looking at preferences over several attributes under uncertainty. It has been 
separately introduced, under different names, by de Finetti (1952) and Richard (1975). The terminology "correlation aversion" comes from Epstein and Tanny (1980). Basically, when considering preferences over bivariate lotteries, correlation aversion tells whether an individual prefers lotteries that exhibit a positive or a negative correlation. Formally, preferences over bivariate lotteries exhibit a positive correlation aversion if and only if, for all $x_{1}, x_{2}, y_{1}, y_{2}$, such that $x_{1}<x_{2}$ and $y_{1}<y_{2}$, the lottery:

$$
\left\{\begin{array} { l l l } 
{ ( x _ { 1 } , y _ { 2 } ) } & { \text { w.p. } } & { \frac { 1 } { 2 } } \\
{ ( x _ { 2 } , y _ { 1 } ) } & { \text { w.p. } } & { \frac { 1 } { 2 } }
\end{array} \text { is preferred to the lottery } \left\{\begin{array}{lll}
\left(x_{1}, y_{1}\right) & \text { w.p. } & \frac{1}{2} \\
\left(x_{2}, y_{2}\right) & \text { w.p. } & \frac{1}{2}
\end{array}\right.\right.
$$

One can define a simple index of correlation aversion with respect to consumption at age $t$ and consumption at age $t+1$ by:

$$
\rho_{t}(z)=\frac{-\frac{\partial^{2}}{\partial z_{t+1} \partial z_{t}} U(z)}{\frac{\partial}{\partial z_{t+1}} U(z)}
$$

for all life such that $z_{t+1} \neq d$. This index is positive (resp. negative) if individuals prefer consumption lotteries at times $t$ and $t+1$ to be negatively (resp. positively) correlated. Moreover, working along the same lines as Bommier (2007), one could relate this index of correlation to the amount of consumption that is necessary to compensate for a positive and infinitesimally small correlation. ${ }^{5}$

The expression that relates ICA to the functions $u$ and $v$ is very simple:

$$
\rho_{t}(z)=v^{\prime}\left(z_{t}\right)
$$

ICA is then equal to zero with the additive specification, and positive when we consider increasing functions $v$.

ICA proves to be an important characteristic of individual preferences in a

\footnotetext{
by

${ }^{5}$ In Bommier (2007), the index of correlation is defined in a slightly different way and given$$
\frac{-\frac{\partial^{2}}{\partial z_{t+1} \partial z_{t}} U(z)}{\frac{1}{2} \frac{\partial}{\partial z_{t}} U(z)+\frac{1}{2} \frac{\partial}{\partial z_{t+1}} U(z)}
$$

Thus the formula of the correlation premium should be modified accordingly. In the limit case where the length of the time period goes to zero, both definitions coincide.
} 
number of instances. For example, Bommier (2007) shows that ICA is simply related to the difference between local measures of relative risk aversion and intertemporal elasticity of substitution. It is thus natural to see ICA playing a major role for determining optimal financial strategies, as shown in Ahn (1989), van der Ploeg (1993), for example.

Still, ICA is probably even more important when accounting for the risk of death. The point is that death is an irreversible event. Thus in the case of death at age $t$, the individual states at ages greater than $t$ are all set to the "death" state. A bad outcome $\left(z_{t}=d\right)$ in period $t$ is thus necessarily combined with a bad outcome in period $\tau>t$. Since ICA reflects the aversion for combining bad outcomes in a given period with bad outcomes in another period, we expect ICA to strongly affect how individuals respond to the risk of death. But, before getting there, we explain how the notion of time preferences and ICA lead to draw a natural partition of the set of stationary preferences.

\section{$7 \quad$ A typology of stationary lifetime preferences}

Let us first express very simple facts that summarize in formal terms what appears from the analytic expressions that were given in the previous section. Some of these facts are well known and are discussed in Keeney and Raiffa (1973) or in Wakker, Jansen and Stiggelbout (2004). Others are fairly obvious. They are formally stated in Results 1 and 2, below, which aim at explaining how simple considerations about time preferences and ICA lead to delimit particular subsets within the set of stationary preferences.

Result 1 The following statements are equivalent:

1. Preferences are additive or multiplicative.

2. Preferences are weakly separable. ${ }^{6}$

3. The rate of time preference is independent of the consumption profile, age and the length of life.

\footnotetext{
${ }^{6}$ Weak separability means that the marginal rate of substitution between consumptions at two different ages is unaffected by consumption at another age.
} 
4. The rate of time preference is constant along any (finite) constant consumption path.

Proof. See Appendix A.

Result 2 1. Preferences are additive if and only if intertemporal correlation aversion always equals zero.

2. Preferences are multiplicative if and only if the rate of time preference always equals zero.

Proof. The first statement immediately follows from equation (8). As for the second statement, we know from (6) that the rate of time preference always equals zero if and only if $u^{\prime}\left(c_{t}\right)\left(1-e^{-v\left(c_{t}\right)}\right)-v^{\prime}\left(c_{t}\right) u\left(c_{t}\right) e^{-v\left(c_{t}\right)}=0$. This equivalent to $u^{\prime}(c) h(c)-u(c) h^{\prime}(c)=0$ where $h$ is the function given by $h(c)=\left(1-e^{-v(c)}\right)$. Under assumption 1, this is the case if and only if $u$ and $h$ are proportional, which, by definition, means that preferences are multiplicative.

A simple picture of the set of stationary preferences follows from these three propositions (see Figure 1).

Result 2 emphasizes that stationary preferences can be represented by a multiplicative utility function if and only if individuals have no pure time preferences. This may seem an unpleasant feature of the multiplicative model, in contradiction with empirical findings indicating that individuals prefer present consumption over future consumption. However, as was first emphasized in Bommier (2006), the combination of ICA and uncertainty can generate sizable time discounting. In particular, when lifetime is random, the model with zero time preferences but positive ICA can generate discount rates that are comparable in size with what it is usually assumed in the economic literature. This result was obtained by focusing on weakly separable specifications, and assuming an infinite value of life. We extend below the analysis to any stationary preferences, including those who combine pure time preferences and ICA, in order to obtain a general decomposition of the rate of time discounting. 


\section{Discount rate and intertemporal correlation aversion with mortality risk}

In problems like that of life cycle consumption planning of human beings, there is ex-ante always considerable uncertainty about the length of life. Therefore, the concepts that are defined for a given length of life (such as those discussed in Section 6) cannot be directly observed. Typically, what can be observed are marginal concepts that describe individual preferences in a neighborhood of non-degenerate lotteries on the length of life. For example, we do not observe individuals' rate of time preference, but how individuals discount consumption knowing that the length of life is uncertain. Also, we do not observe the marginal rate of substitution between length of life and consumption, but the willingness to pay for reducing the hazard risk of death at a given moment in time. The aim of this section is to make explicit the link between time preferences, ICA and what can be inferred from the behavior of agents with an uncertain lifetime.

In the following, a mortality pattern, that we will denote by the letter $\mu$, will be a sequence of age specific mortality rates $\mu=\left(\mu_{t}\right)_{t \in \mathbb{N}}$. For all $t \geq 0, \mu_{t} \in[0,1]$ is the probability of dying at the end of period $t$, conditional on being alive at the beginning of period $t$. It will be assumed that for all mortality patterns considered there exists some finite time $\tau$ such that $\mu_{\tau}=1$. To any profile of mortality rates corresponds a distribution of the age at death $\pi_{t}$ and a survival profile $s_{t}$. The number $\pi_{t}$ gives the probability that the agent dies at the end of period $t$. We have $\pi_{0}=\mu_{0}$ and for $t>0$ :

$$
\pi_{t}=\mu_{t} \prod_{i=0}^{t-1}\left(1-\mu_{i}\right)
$$

The number $s_{t}$ gives the probability that an individual will be alive during period

$t$. We have $s_{0}=1$ and for $t>0$ :

$$
s_{t}=\prod_{i=0}^{t-1}\left(1-\mu_{i}\right)
$$

Because we assume that there is always some finite time $\tau$ such that $\mu_{\tau}=1$ the 
sequences $s_{t}$ and $\pi_{t}$ will be equal to zero after a finite time. In practice, this guarantees that all the infinite sums we consider below are well defined. Note that we have the simple relations:

$$
\pi_{t}=s_{t}-s_{t+1}
$$

which also implies:

$$
s_{t}=\sum_{i=t}^{+\infty} \pi_{i}
$$

In the case where agents face an uncertain lifetime, they make choices that will generate different "lives" (i.e. different elements of $Z$ ) depending on when they die. For example, they may have to choose a consumption profile $c \in \mathbb{R}_{+}^{\mathbb{N}}$ which, in case of death at age $T$, will generate a life $z \in Z$ given by:

$$
z_{i}=c_{i} \text { for all } i \leq T \text { and } z_{i}=d \text { for all } i>T
$$

For practicality we will denote such a life by $(c, T)$.

For any real function $f(x, T)$ that depends on some attributes, $x$, and on the age at death, $T$, we define the $\mu, t$-average of $f(x, T)$ that we denote $E_{\mu, t} f(x,$.$) ,$ by:

$$
E_{\mu, t} f(x, .)=\frac{\sum_{i=t}^{+\infty} \pi_{i} f(x, i)}{\sum_{i=t}^{+\infty} \pi_{i}}
$$

$E_{\mu, t} f(x,$.$) is simply the average of f(x, T)$ when $T$ follows the distribution of the age at death truncated at $T \geq t$. For example, in the case where $f(x, T)$ is the age at death (that is when $f(x, T)=T$ ), then $E_{\mu, t} f(x,$.$) is the average age at$ death of the individuals that were alive at the beginning of period $t$.

Combining (9), (10) and (11), one readily obtains:

$$
E_{\mu, t} f(x, .)=f(x, t)+\sum_{i=t+1}^{+\infty} \frac{s_{i}}{s_{t}}[f(x, i)-f(x, i-1)]
$$

so that the $\mu, t$-average of $f(x, T)$ can be expressed as a function of the survival probabilities, instead of a function of the distribution of the age at death.

Life cycle planning involves choosing a consumption path $c \in \mathbb{R}_{+}^{\mathbb{N}}$, knowing 
that, in the case death occurs at age $T$, this will generate the life $(c, T)$ and a utility:

$$
U(c, T)=\sum_{i=0}^{T} u\left(c_{i}\right) \exp \left(-\sum_{j=0}^{i-1} v\left(c_{j}\right)\right)
$$

When $T$ is random, individuals aim at maximizing the expected utility, given the distribution of $T$, that is $E_{\mu, 0} U(c,$.$) given by:$

$$
E_{\mu, 0} U(c, .)=\sum_{T=0}^{+\infty} \pi_{T} U(c, T)
$$

Using (12), this can be rewritten as:

$$
\begin{aligned}
E_{\mu, 0} U(c, .) & =u\left(c_{0}\right)+\sum_{i=1}^{+\infty} s_{i}[U(c, i)-U(x, i-1)] \\
& =\sum_{i=0}^{+\infty} s_{i} u\left(c_{i}\right) \exp \left(-\sum_{j=0}^{i-1} v\left(c_{j}\right)\right)
\end{aligned}
$$

We recognize here a generalization -in discrete time- of the lifetime utility function suggested by Yaari (1965), which is obtained when $v$ is constant.

Local properties of individual preferences in the neighborhood of elements of $L(Z)$ characterized by a given consumption profile and a non-degenerate mortality pattern can then be defined. For any mortality pattern and any consumption profile, we define the $\mu$-rate of discount at time $t$ by:

$$
R T P_{\mu, t}(c)=1-\left.\frac{\frac{\partial}{\partial c_{t+1}} E_{\mu, 0} U(c, .)}{\frac{\partial}{\partial c_{t}} E_{\mu, 0} U(c, .)}\right|_{c_{t+1}=c_{t}}
$$

the $\mu$-intertemporal correlation aversion at time $t$ by:

$$
\rho_{\mu, t}(c)=\frac{-\frac{\partial^{2}}{\partial c_{t+1} \partial c_{t}} E_{\mu, 0} U(c, .)}{\frac{\partial}{\partial c_{t+1}} E_{\mu, 0} U(c, .)}
$$

and the value of a statistical life at age $t$ by:

$$
V S L_{\mu, t}(c)=-\frac{\frac{\partial}{\partial \mu_{t}} E_{\mu, 0} U(c, .)}{\frac{\partial}{\partial c_{t}} E_{\mu, 0} U(c, .)} .
$$


Equations (15) and (16) extend the definitions given by (5) and (7) to the case where the length of life is not known with certainty. The value of a statistical life, $V S L_{\mu, t}$, is nothing other than the opposite of the marginal rate of substitution between mortality at time $t$ and consumption at time $t$. The terminology Value of a Statistical Life is consistent with that used in Johansson (2002).

We can now express the following result:

Proposition 2 For any consumption profile, $c$, any mortality pattern, $\mu$, and any time, $t$, we have

$$
\begin{aligned}
\rho_{\mu, t}(c) & =\rho_{t}(c, T) \text { for all } T>t \\
R T P_{\mu, t} & =\frac{1}{E_{\mu, t}\left[\frac{1}{R T P_{t}(c, .)}\right]}+\mu_{t}+\mu_{t} \rho_{t} V S L_{\mu, t}(c)-\mu_{t} \frac{1}{E_{\mu, t}\left(\frac{1}{R T P_{t}(c, .)}\right)}
\end{aligned}
$$

\section{Proof. See Appendix B.}

The first part of Proposition 2, which is a consequence of the recursive structure of the expected utility function (14), indicates that the $\mu$-ICA age $t$ is simply equal to the ICA when life duration is certain, and greater than $t$. Observation of ICA is therefore not complicated by the presence of mortality. Direct estimations of ICA can be achieved through simple experiments, as with those developed by Andersen, Harrison, Lau and Ruström (2011). The same statement is not true however for the rate of time preference.

We can observe from equation (18) that the $\mu$-rate of discount is the sum of four terms. The first one, $\frac{1}{E_{\mu, t}\left(\frac{1}{R T P_{t}}\right)}$, is the harmonic mean of the rate of time preference. This term accounts for individuals' pure time preferences. In the case of the additive or multiplicative model, the rate of time preference is a constant and the first term is simply the exogenous rate of time preference. However, in the general case, the rate of time preference may depend on the length of life. Equation (18) indicates that its harmonic mean is what matters when the length of life is random.

The second term is the mortality rate. It accounts for the fact that mortality creates a risk on future consumption. Consumption only occurs in case of survival.

The third term stresses the role of ICA. It vanishes when ICA equals zero, 
and consequently has remained unnoticed in the economic literature that relies on time additive models to account for lifetime uncertainty.

Two parallel lines of argument can be followed to provide intuition about the origin of this third term. The first one sticks to the crude meaning of ICA. Mortality generates a risk on tomorrow's consumption that is positively correlated to a much greater risk: the risk of losing life for ever. If tomorrow's consumption proves to be impossible, because of death, so will be the case of consumption at any date after tomorrow. Agents with positive ICA will react to this correlation by decreasing the risk on tomorrow's consumption: that is by consuming more today and less tomorrow. The magnitude of the reaction depends on the mortality risk, ICA and the value of the other items at risk (life, whose value is given by $\left.V S L_{\mu, t}\right)$. This explains the structure of this third term.

Another way of thinking about the third term of (18) involves considering the (ex-post) distribution of lifetime utility. Mortality makes lifetime utility random: in the standard situation where $u(c(t))$ remains positive, lifetime utility is low in case of an early death, and high in case of a late death. However, reallocating consumption towards early periods of life is a way to make the distribution of lifetime utility less unequal. By consuming early in the life cycle, one increases lifetime utility of short lives and lowers the utility gap between short and long lives. The willingness to reallocate consumption for that purpose depends on individuals' ICA. It vanishes when individuals are indifferent to intertemporal correlation (that is in the additive model) and increases with ICA.

The fourth term in equation (18) is an interaction term between mortality and pure time preference. This term appears because we work with discrete time, but would disappear in continuous time. In fact, when the length of the time period goes to zero, all the first three terms decrease proportionally to the length of the time period. For example if mortality rate at some age is $x \%$ per year -with $x$ small- and we shift to monthly periods, we roughly get a mortality rate of $\frac{x}{12} \%$ per month. The same is true with the rate of time discounting. The fourth term, which is the negative of the product of the mortality rate and the harmonic mean of the rate of time discounting, would decrease -in absolute value- proportionally to the square of the length of the time period. Shifting from a yearly time period 
to a monthly one, would lead to divide this term by 144 , while the others would be divided by 12 only. As the length of the time period tends towards zero, this second-order term becomes negligible; it eventually disappears in the continuous setting.

If time discounting may be only partially driven by time preferences, the key question is how, in theory at least, we could identify individuals' pure time preferences. Looking at the role of mortality in Proposition 2 provides a solution. Indeed, from equation (18), if we consider that the length of the time periods are small enough so that second-order terms can be neglected, we obtain that the rate of time discounting at age $t$ is a linear function of $\mu_{t}$, with slope $\left(1+\rho_{t} V S L_{\mu, t}\right)$ and intercept $\frac{1}{E_{\mu, t}\left(\frac{1}{R T P_{t}}\right)} \cdot{ }^{7}$ In absence of pure time preferences the elasticity of the $\mu$-rate of discount at age $t$ with respect to mortality at age $t$ equals 1 . If individuals have positive pure time preferences then this elasticity is smaller than one. More generally, a regression of the rate of discount at age $t$ with a list of variables including the mortality rate at age $t$ could theoretically provide estimates of both $E_{\mu, t}\left(\frac{1}{R T P_{t}}\right)$ and $\rho_{t} V S L_{\mu, t}$. The difficulty of the task should not be underestimated, however, since one needs to control for mortality rates at ages greater than $t$, which are strongly correlated with the mortality rate at age $t$. Following this direction would thus require us to have simultaneous estimates on individual rates of discount, on the one hand, and mortality rates at all (present and future) ages, on the other hand.

Proposition 2 makes it clear that ICA plays a central role when considering the impact of mortality. In the case where ICA is neglected (or assumed to equal zero) then equation (18) reduces to $R T P_{\mu, t}=R T P+\mu_{t}(1-R T P) .^{8}$ In that case, mortality does contribute to the rate of time discounting, but, quantitatively

\footnotetext{
${ }^{7}$ Interpretation of equation (18) requires to account for the fact $V S L_{\mu, t}$ and $E_{\mu, t}\left(\frac{1}{R T P_{t}}\right)$ also depend on mortality at age $t$. However, as is explained at the end of Appendix $\mathrm{B}$, where the role of $\mu_{t}$ is made fully explicit, this dependence only generates second order terms that can be neglected if we assume that the length of the time period is small.

${ }^{8}$ In the case where agents have zero ICA, preferences are additive and the rate of time preference is a constant. In the particular case where $R T P=0$, then the rate of time discounting equals the mortality rate. Assuming constant mortality, no exogenous time preference $(R T P=$ $0)$ and no ICA $\left(\rho_{t}=0\right)$ could then be a way to provide suppport for the additive model with exponential discounting. This was noted in Mas-Colell, Whinston and Green (1995, p. 734), with no explicit mention of the assumption of intertemporal correlation neutrality, however.
} 
speaking, when considering human mortality, the contribution is small unless considering people at old ages or with low life expectancy. Indeed, in a country like the US, average mortality rates equal $0.1 \%$ per year at 40 and do not reach $1 \%$ per year before age 62 , and are therefore small compared to usual estimates for the rate of discount. When agents have a positive ICA (that is: $\rho_{t}>0$ ), there is an another term, $\mu_{t} \rho_{t} V S L_{\mu, t}(c)$, through which mortality contributes to time discounting. This term can generate substantial time discounting, even if mortality and ICA are small, since the value of statistical life is usually estimated to be very large. ${ }^{9}$

\section{Life cycle consumption}

Allowing for non zero ICA significantly changes the predictions of life cycle models and provides new insights on a number of topics. As mentioned in Section 2, applications bearing on the impact of mortality decline on aggregate savings, the value of life, the design of social security systems and on life-cycle portfolio choices are developed in other papers. We shall not return to these aspects, but focus on a simple issue related to consumption smoothing.

There is an on-going debate on the shape of the life cycle consumption profile, and how this can be rationalized. It is indeed found that the relation between age and consumption has an inverse U-shape, consumption increasing during the first part of the lifecycle and then declining. There is of course some heterogeneity in the estimates of this consumption profile, and at which age consumption is peaking, but the inverse U-shape relationship is quite robust. The literature also agrees that variation in household composition may partly - but not completelyaccount for the age variation of consumption. In order to have a plausible explanation, while relying on the standard additive life cycle model, one has to incorporate numbers of features, including imperfect (or inexistent) annuity markets and the need for precautionary savings. The particular role of mortality is the object of Feigenbaum (2008), who explains that, in the absence of annuity mar-

\footnotetext{
${ }^{9}$ The priceless life context approximation suggested in Bommier (2006) is a limit case where ICA tends to zero (preferences being almost linear) and the VSL goes to infinity, the product of the two having a finite limit.
} 
kets, mortality can indeed generate a hump shape consumption profile, but which does not match what is documented by empirical papers. Feigenbaum actually concludes that "mortality is an unlikely answer to the question of consumption hump" (Feigenbaum, 2008, p. 846).

The object of the current section is to discuss whether departing from the additive specification, and opting for a model that allows for ICA, may provide an explanation for the shape of the consumption profile which was estimated empirical studies. More precisely, we will investigate whether the additive and the multiplicative models may generate consumption profiles that match the one estimated by Fernández-Villaverde and Krueger (2007). ${ }^{10}$

In practice we consider the 1990 (male-female average) US mortality rates, ${ }^{11}$ downloaded from the Human Mortality Database, and look at the optimal consumption profile of agents who have to allocate some exogenous wealth endowment having access to perfect credit and annuity markets. We do not model labor supply and/or retirement behavior. Though in presence of perfect credit markets, agents face a single budget constraint (expected lifetime consumption should not exceed expected lifetime earnings) and are therefore confronted by a problem which is very similar to the one we consider.

Given initial wealth (or lifetime earnings), $\Omega_{0}$, agents have to choose a consumption profile solving:

$$
\max E_{\mu, 0} U(c, .) \text { subject to } \sum_{t=0}^{+\infty} s_{t} R^{-t} c_{t} \leq \Omega_{0}
$$

where $R$ is the gross return of the risk-free asset. The budget constraint accounts for survival probabilities since, in absence of bequest motives, agents should invest all their wealth in annuities, which provide a return $\frac{R}{1-\mu_{t}}$ in case of survival,

\footnotetext{
${ }^{10}$ Our choice to use the results of Fernández-Villaverde and Krueger (2007) as a reference is partly due to the fact they provide estimates until old ages, contrary to many other studies that focus on what happen at younger ages.

${ }^{11}$ Estimates from Fernández-Villaverde and Krueger come from a US data gathered during the years 1980-2001. This is why why we use 1990 US mortality data.
} 
exceeding the return of the risk free asset, $R .^{12}$

We solve this problem for both the additive and multiplicative specification. We therefore compare what is obtained when assuming:

$$
U(c, T)=\sum_{i=0}^{T} \beta^{i} u\left(z_{i}\right) \text { (additive case) }
$$

with what is obtained when:

$$
U(c, T)=\frac{1}{k}\left(1-\exp \left(-k \sum_{i=0}^{T} w\left(z_{i}\right)\right)\right) \quad(\text { multiplicative case })
$$

For both the additive and multiplicative specifications we assume that agents have a constant intertemporal elasticity of substitution. This means that $-\frac{u^{\prime}(c)}{c u^{\prime \prime}(c)}$ and $-\frac{w^{\prime}(c)}{c w^{\prime \prime}(c)}$ should be independent of $c$ or, equivalently,

$$
u(c)=u_{0}+\frac{c^{1-\gamma_{u}}}{1-\gamma_{u}} \text { and } w(c)=w_{0}+\frac{c^{1-\gamma_{w}}}{1-\gamma_{w}}
$$

for some constants $u_{0}, w_{0}, \gamma_{u}$ and $\gamma_{w}$. The constants $u_{0}$ and $w_{0}$ cannot be normalized to zero, as representations (3) and (4) already assume a specific normalization for the functions $u$ and $w$, which must fulfill $u(d)=w(d)=0$. These constants $u_{0}$ and $w_{0}$ impact the welfare gap between being dead and being alive and consuming $c$. As such they are the main determinants of the value of a statistical

${ }^{12}$ The period budget contraints can be written:

$$
\begin{aligned}
& \Omega_{t+1}=\frac{R}{\left(1-\mu_{t}\right)}\left(\Omega_{t}-c_{t}\right) \\
& \Omega_{t+1} \geq 0
\end{aligned}
$$

By iteration we get:

$$
\Omega_{0}=c_{0}+\sum_{t=1}^{n}\left(\prod_{i=0}^{t-1} \frac{\left(1-\mu_{i}\right)}{R}\right) c_{t}+\left(\prod_{i=0}^{n} \frac{\left(1-\mu_{i}\right)}{R}\right) \Omega_{n+1}
$$

which taking the limit $n \rightarrow \infty$ provides

$$
\sum_{t=0}^{+\infty} s_{t} R^{-t} c_{t} \leq \Omega_{0}
$$

as stated in (19). 
life, for which we have empirical estimates to calibrate our models. However, as the value of a statistical life also depends on other parameters (and in particular of $\beta, k, \gamma_{u}$ and $\gamma_{w}$ ), calibrations of the additive and multiplicative models have to be performed separately leading to different values for $u_{0}$ and $w_{0}$.

In practice we will choose $u_{0}$ and $w_{0}$ so that an individual having a background mortality provided by the 1990 US life table and income 1 would be indifferent between facing an extra risk of death of 0.0001 per year, from age 20 till the end of his/her life, or having an income of 1.035. This is consistent with a (survival weighted) average value of a statistical life of 7 million dollars (a reasonable value, according to Viscusi and Aldy, 2003) if we assume that one unit of income actually corresponds to 20000 dollars per year.

The parameters $\beta, k, \gamma_{u}$ and $\gamma_{w}$ are chosen to minimize the (survival weighted) average distance between the predicted consumption profile and the one provided in figure 4 of Fernández-Villaverde and Krueger (2007).

In the case of the additive model it is well known that the solution to the optimization problem (19) involves choosing a consumption profile such that

$$
c_{t}=c_{0}\left(\frac{R}{\beta}\right)^{\frac{t}{\gamma_{u}}}
$$

Thus all combinations of the parameters that give the same value for $\left(\frac{R}{\beta}\right)^{\frac{1}{\gamma_{u}}}$ will provide the same solution. There is therefore an indeterminacy when trying to find the best fit with the profile taken from Fernández-Villaverde and Krueger (2007). For simplicity, we shall therefore report the value of the optimal consumption growth rate, denoted $g$ (the one which best fits the data), knowing that for any $\gamma_{u}$ it can be obtained by setting $\beta$ equal to $R(1+g)^{-\gamma_{u}}$.

As for the multiplicative model, the optimization problem is complex, the expected utility function being non linear. No analytic solution can be provided and the results rely on numerical optimization.

Figure 2 plots the estimates from Fernández-Villaverde and Krueger (2007) as well as the predictions obtained when using the additive and multiplicative models, assuming $R-1=2.5 \%$ per year. The additive model which constrains 
consumption to be monotonic is unable to provide a good fit or reproduce the hump-shape profile of consumption. The rate of consumption growth which minimizes the average distance with Fernández-Villaverde's and Krueger's result is $g=-0.25 \%$ per year, obtained for example with $\gamma_{u}=1$ and $\beta-1=2.76 \%$ per year. The multiplicative model provides a much better fit, although in order to match the data quite closely one has to assume a relatively high elasticity of substitution, $\frac{1}{\gamma_{w}}$, equal to 1.41 . We shall note however that there are several studies including Vissinng-Jorgensen and Attanasio (2003) and Andersen, Harrison, Lau and Ruström (2011) -both allowing for ICA- that estimate intertemporal elasticity of substitution in that range. In order to provide some idea of the our explanation's potential, we also run simulation where we constrain the elasticity of substitution to equal 1. For the multiplicative model, we still find a non-monotonic consumption profile, though with a smaller curvature than that of Fernández-Villaverde and Krueger (Figure 3).

Accounting for ICA may thus help to explain the hump-shape of the observed consumption profile. This should not be taken to mean that ICA and mortality might be the single explanation for the hump shape of consumption. In fact, there are some aspects, such as the low level of consumption before age 30, that models with ICA have difficulty in predicting, while they could simply result from liquidity constraints. The potential role of ICA should not be considered as a substitute for previous explanations based on market imperfections or precautionary savings but rather as being complementary.

It is worth emphasizing that preferences being stationary, the only source of variations in the predicted consumption growth rate shown in Figures 2 and 3 is the positive relation between age and mortality. This positive relation is what makes life expectancy shrink as an individual is getting older. In that sense, the hump shape of consumption obtained with the multiplicative model reflects a pure life cycle effect. We shall also note that the additive and multiplicative models, which are two extensions of the simplest model that assumes both a zero ICA and a zero rate of time preference, have the same number of degrees of freedom. Thus, if one turns out to have a better predictive power than the other, this is not the result of a greater complexity, but simply the consequence 
of different underlying assumptions.

\section{Conclusion}

A general representation of stationary preferences was provided. Some particular specifications were also underlined as corresponding to additional assumptions on individual preferences (Figure 1). This naturally suggests several candidates for lifetime utility function. The simplest choice, and also the most restrictive, involves assuming that individuals have neither pure time preferences nor ICA. Two natural and symmetrical extensions then seem possible: one introducing pure time preferences (which gives the additive model) and another one introducing ICA (as in the multiplicative model). Pure time preferences and ICA may eventually be combined, as in the general recursive form.

The literature on intertemporal choice contrasts with the symmetric picture we drew. While the additive model has been extensively considered, and used as a basis for extensions that do not lie within the general framework we consider, the multiplicative model remained unexploited.

There are plenty of historical and technical reasons that could explain why life cycle theory did not explore the case of multiplicative preferences. In particular, the multiplicative model is an attractive option only when both the finiteness and the randomness of the length of life are accounted for. Therefore it could not emerge from studies that considered that the length of life was infinite or known in advance. Moreover, although the multiplicative model is as simple as the additive one in terms of degrees of freedom, it is mathematically speaking less tractable. For example, while it is extremely simple to analytically derive the shape of the optimal consumption in the additive case (with perfect markets), it is impossible to find an explicit solution in the multiplicative case. The solution is closely related to mortality rates and can only be numerically estimated.

Opting for a mathematically convenient theory has an undisputable advantage: it makes economists' lives easier. But the cost may be high. There are many aspects of human behavior that are difficult to explain with the additive model, while they seem rather natural when accounting for ICA. We discussed 
above a fact that was identified as an "empirical puzzle": the hump-shape of life cycle consumption profiles. But there are other issues that seem as important. We end the paper by reviewing three of them.

The first one concerns the heterogeneity in discount rates. Women, rich people and non-whites (in the USA) are usually found to be significantly less impatient than men, poor people and whites, the difference in discount rates exceeding that in mortality rates. With the additive model, such heterogeneity can only result from heterogeneity in pure time preferences. Thus, the dominant interpretation is that heterogeneity in impatience reflects fundamental differences in taste, whose origins lie deep in human nature or cultural constructs. Men and women, rich and poor, whites and non-whites would simply have different rationalities. Conversely, a life cycle theory that accounts for ICA would explain a great part of the heterogeneity in discount rates by the heterogeneity in mortality. Women and men, rich and poor, etc. could allocate resources over time differently not because they have different preferences, but simply because of their mortality. As argued in Peart and Levy (2003), these diverging interpretations may be used to support fundamentally different ideologies.

The second issue concerns the effect of mortality changes. The huge decline in mortality rates observed over the last two centuries, as well as the dramatic increase of mortality observed in the regions severely touched by the AIDS epidemic, are about the most significant events in recent human history. Naturally, several papers have studied the economic impact of mortality changes. ${ }^{13}$ But almost all of them rely on the additive model. Accounting for ICA would radically modify our view of the effect of mortality changes and indirectly our understanding of economic development.

The last issue concerns the amount of resources that should be dedicated to increase longevity. This is a central question in our society where medical expenses are rising very rapidly. Again, as discussed in Bommier and Villeneuve (2010), the dominant approach in the value of life literature involves using the additive model, while accounting for ICA would significantly improve the capacity

\footnotetext{
${ }^{13}$ See for example Bloom, Canning and Graham (2003), Boucekkine, de la Croix and Licandro (2003), Cavalcanti Ferreira and Pessôa (2003) and Becker, Philipson and Soares (2005).
} 
of the theory to fit empirical data and suggest very different policy guidance.

ICA is a key element for modelling intertemporal choice under uncertainty. The reason is that in many cases, agents are facing risks which have durable consequences (such as the risk of death, but also the risk of bankruptcy, etc. ). The time structure then ends up generating a strong positive correlation between what may happen at different periods of time, so that aversion for correlation appears to be central for evaluating these risks. The recursive specification obtained in Proposition 1 and, in particular, the multiplicative model provide simple ways to account for ICA.

\section{A Proof of Result 1}

Using (3) and (4), it is straightforward to check that $1 \Rightarrow 2$. Moreover, $1 \Rightarrow 3$ follows from (6). 4 is explicitly weaker than 3 and, therefore, $3 \Rightarrow 4$.

Let us now prove that $2 \Rightarrow 1$. Denote:

$A=\left\{c \in \mathbb{R}_{+} \mid v^{\prime}(c)=0\right\}$ and $M=\left\{c \in \mathbb{R}_{+} \mid u^{\prime}\left(c_{t}\right)\left(1-e^{-v\left(c_{t}\right)}\right)-v^{\prime}\left(c_{t}\right) u\left(c_{t}\right) e^{-v\left(c_{t}\right)}=0\right\}$

By definition (and because of Assumption 1) preferences are additive if and only if $A=\mathbb{R}_{+}$and multiplicative if and only if $M=\mathbb{R}_{+}$. The rate of time preference at time $t$ is given by:

$$
R T P_{t}=\frac{u^{\prime}\left(z_{t}\right)\left(1-e^{-v\left(z_{t}\right)}\right)-v^{\prime}\left(z_{t}\right) u\left(z_{t}\right) e^{-v\left(z_{t}\right)}}{u^{\prime}\left(z_{t}\right)-v^{\prime}\left(z_{t}\right) \sum_{i=t+1}^{+\infty} u\left(z_{i}\right) \exp \left(-\sum_{j=t}^{i-1} v\left(z_{j}\right)\right)}
$$

and therefore:

$$
2 \Rightarrow \text { for any } t_{1} \neq t \text { we have } \frac{\partial R T P_{t}}{\partial z_{t_{1}}}=0
$$


But, by derivation of $(6)$, for $t_{1}>t$, such that $z_{t_{1}} \neq d$ we find that

$$
\frac{\partial R T P_{t}}{\partial z_{t_{1}}}=0 \Leftrightarrow\left(\begin{array}{c}
u^{\prime}\left(z_{t}\right)\left(1-e^{-v\left(z_{t}\right)}\right)-v^{\prime}\left(z_{t}\right) u\left(z_{t}\right) e^{-v\left(z_{t}\right)}=0 \\
\text { or } \\
v^{\prime}\left(z_{t}\right)=0 \\
\text { or } \\
\frac{\partial}{\partial z_{t_{1}}}\left(\sum_{i=t+1}^{+\infty} u\left(z_{i}\right) \exp \left(-\sum_{j=t}^{i-1} v\left(z_{j}\right)\right)\right)=0
\end{array}\right)
$$

Assumption 2 implies that:

$$
\frac{\partial}{\partial z_{t_{i}}}\left(\sum_{i=t+1}^{+\infty} u\left(z_{i}\right) \exp \left(-\sum_{j=t}^{i-1} v\left(z_{j}\right)\right)\right) \neq 0 \text { for all } t_{1}>t
$$

Thus, it is clear that $\frac{\partial R T P_{t}}{\partial z_{t_{1}}}=0$ if and only if $u^{\prime}\left(z_{t}\right)\left(1-e^{-v\left(z_{t}\right)}\right)-v^{\prime}\left(z_{t}\right) u\left(z_{t}\right) e^{-v\left(z_{t}\right)}=$ 0 or $v^{\prime}\left(z_{t}\right)=0$. Therefore:

$$
2 \Rightarrow A \cup M=\mathbb{R}_{+}
$$

We now prove that $A \cup M=\mathbb{R}_{+}$, together with Assumptions 1 and 2, implies that $A=\emptyset$ (and $M=\mathbb{R}_{+}$) or $A=\mathbb{R}_{+}$.

Denote $\complement_{A}$ the complement of $A$. Because of Assumption $1, \complement_{A}$ is open. Assume that $\complement_{A}$ is not empty and consider $c^{*} \in \complement_{A}$. Note $I_{c}$ the largest open interval that is included in $\complement_{A}$ and contains $c^{*}$. Since $A \cup M=\mathbb{R}_{+}$we know that $\complement_{A} \subset M$ and $I_{c} \subset M$. Thus, there must exist a constant $k_{I}$ such that $\left(1-e^{-v(c)}\right)=k_{I} u(c)$ for all $c \in I_{c}$. This implies that, $v^{\prime}(c) e^{-v\left(z_{t}\right)}=k_{I} u^{\prime}(c)$. By continuity (Assumption 1 ), such a relation must extend to $\overline{I_{c}}$, the closure of $I_{c}$. Moreover, the constant $k_{I}$ is necessarily different from zero (by definition of $A$ ) and, with Assumption 2, this implies that $\overline{I_{c}} \subset \complement_{A}$ (otherwise there would exist $c \in \mathbb{R}_{+}$such that $v^{\prime}(c)=u^{\prime}(c)=0$ which would contradict $\left.(20)\right)$. But as $I_{c}$ is the largest open interval of $\complement_{A}$ that contains $c^{*}$, it is necessarily the case that $\overline{I_{c}} \subset I_{c}$. Therefore $I_{c}=\mathbb{R}_{+}$and $\complement_{A}=\mathbb{R}_{+}$. Thus

$$
\left(A \cup M=\mathbb{R}_{+}\right) \Rightarrow 1
$$

It only remains to prove that $4 \Rightarrow 1$. Lets consider a life composed of constant 
path at a level $c$, till an age $T$, after which the agent is dead. From (6) we have for all $t<T$ :

$$
R T P_{t}=\frac{u^{\prime}(c)\left(1-e^{-v(c)}\right)-v^{\prime}(c) u(c) e^{-v(c)}}{u^{\prime}(c)-v^{\prime}(c) u(c) \sum_{i=t+1}^{T} \exp \left(-\sum_{j=t}^{i-1} v(c)\right)}
$$

Note that

$$
\sum_{i=t+1}^{T} \exp \left(-\sum_{j=t}^{i-1} v(c)\right)-\sum_{i=t+2}^{T} \exp \left(-\sum_{j=t+1}^{i-1} v(c)\right)=\exp \left(-\sum_{j=t}^{T-1} v(c)\right) \neq 0
$$

Thus $R T P_{t}=R T P_{t+1}$ if and only if $u^{\prime}(c)\left(1-e^{-v(c)}\right)-v^{\prime}(c) u(c) e^{-v(c)}=0$ or $u(c) v^{\prime}(c)=0$. In other words $4 \Rightarrow A \cup M \cup\{c \mid u(c)=0\}=\mathbb{R}_{+}$. Note that there exists at most one point $c$ such that $u(c)=0$ (otherwise Assumption 2 would not be fulfilled). Since $A \cup M$ is closed we have $A \cup M \cup\{c \mid u(c)=0\}=\mathbb{R}_{+} \Rightarrow$ $A \cup M=\mathbb{R}_{+}$. Using (21), we find that $4 \Rightarrow 1$.

\section{B Proof of Proposition 2}

By derivation of equation (14), we obtain:

$$
\frac{\partial}{\partial c_{t}} E_{\mu, 0} U(c)=s_{t} \exp \left(-\sum_{j=1}^{t-1} v\left(c_{j}\right)\right)\left[u^{\prime}\left(c_{t}\right)-v^{\prime}\left(c_{t}\right) \sum_{i=t+1}^{+\infty} \frac{s_{i}}{s_{t}} u\left(c_{i}\right) \exp \left(-\sum_{j=t}^{i-1} v\left(c_{j}\right)\right)\right]
$$

For $t>1$, taking the derivative with respect to $c_{t-1}$ leads to:

$$
\frac{\partial^{2}}{\partial c_{t-1} \partial c_{t}} E_{\mu, 0} U(c)=-v^{\prime}\left(c_{t-1}\right) \frac{\partial}{\partial c_{t}} E_{\mu, 0} U(c)
$$

Or again

$$
\rho_{\mu, t-1}(c)=-v^{\prime}\left(c_{t-1}\right)=\rho_{t-1}(c, T) \text { for all } T>t-1
$$

Equation (17) is just a reformulation of this result, with the index $t-1$ replaced by $t$.

In order to obtain equation (18), let us first compute the expression of $V S L_{\mu, t}(c)$. From: 


$$
s_{i}=\prod_{j=1}^{i-1}\left(1-\mu_{i}\right)
$$

one obtains:

$$
\begin{aligned}
& \frac{\partial s_{i}}{\partial \mu_{t}}=0 \text { if } t \geq i \\
& \frac{\partial s_{i}}{\partial \mu_{t}}=-\frac{s_{i}}{\left(1-\mu_{t}\right)} \text { if } t<i
\end{aligned}
$$

Thus:

$$
\frac{\partial}{\partial \mu_{t}} E_{\mu, 0} U(c)=-\frac{1}{\left(1-\mu_{t}\right)} \sum_{i=t+1}^{+\infty} s_{i} u\left(c_{i}\right) \exp \left(-\sum_{j=1}^{i-1} v\left(c_{j}\right)\right)
$$

Using $(22)$ and $s_{t+1}=\left(1-\mu_{t}\right) s_{t}$, we obtain:

$$
V S L_{\mu, t}=\frac{\sum_{i=t+1}^{+\infty} \frac{s_{i}}{s_{t+1}} u\left(c_{i}\right) \exp \left(-\sum_{j=1}^{i-1} v\left(c_{j}\right)\right)}{\left[u^{\prime}\left(c_{t}\right)-v^{\prime}\left(c_{t}\right) \sum_{i=t+1}^{+\infty} \frac{s_{i}}{s_{t}} u\left(c_{i}\right) \exp \left(-\sum_{j=1}^{i-1} v\left(c_{j}\right)\right)\right]}
$$

We now have all elements to derive (18). From (22), applied in $t$ and in $t+1$, we have:

$$
\left.\frac{\frac{\partial}{\partial c_{t+1}} E_{\mu, 0} U(c)}{\frac{\partial}{\partial c_{t}} E_{\mu, 0} U(c)}\right|_{c_{t+1}=c_{t}}=\frac{s_{t+1}}{s_{t}} e^{-v\left(c_{t}\right)} \frac{u^{\prime}\left(c_{t}\right)-v^{\prime}\left(c_{t}\right) \sum_{i=t+2}^{+\infty} \frac{s_{i}}{s_{t+1}} u\left(c_{i}\right) \exp \left(-\sum_{j=t+1}^{i-1} v\left(c_{j}\right)\right)}{u^{\prime}\left(c_{t}\right)-v^{\prime}\left(c_{t}\right) \sum_{i=t+1}^{+\infty} \frac{s_{i}}{s_{t}} u\left(c_{i}\right) \exp \left(-\sum_{j=t}^{i-1} v\left(c_{j}\right)\right)}
$$

Or again:

$$
\left.\frac{\frac{\partial}{\partial c_{t+1}} E_{\mu, 0} U(c)}{\frac{\partial}{\partial c_{t}} E_{\mu, 0} U(c)}\right|_{c_{t+1}=c_{t}}=\frac{s_{t+1}}{s_{t}} e^{-v\left(c_{t}\right)} \frac{u^{\prime}\left(c_{t}\right)-v^{\prime}\left(c_{t}\right) \sum_{i=t+2}^{+\infty} \frac{s_{i}}{s_{t+1}} u\left(c_{i}\right) \exp \left(-\sum_{j=t+1}^{i-1} v\left(c_{j}\right)\right)}{u^{\prime}\left(c_{t}\right)-v^{\prime}\left(c_{t}\right) \sum_{i=t+1}^{+\infty} \frac{s_{i}}{s_{t}} u\left(c_{i}\right) \exp \left(-\sum_{j=t}^{i-1} v\left(c_{j}\right)\right)}
$$




$$
\begin{aligned}
= & \frac{s_{t+1}}{s_{t}} \frac{u^{\prime}\left(c_{t}\right) e^{-v\left(c_{t}\right)}+v^{\prime}\left(c_{t}\right) u\left(c_{t}\right) e^{-v\left(c_{t}\right)}}{u^{\prime}\left(c_{t}\right)-v^{\prime}\left(c_{t}\right) \sum_{i=t+1}^{+\infty} \frac{s_{i}}{s_{t}} u\left(c_{i}\right) \exp \left(-\sum_{j=t}^{i-1} v\left(c_{j}\right)\right)} \\
& -\frac{s_{t+1}}{s_{t}} \frac{v^{\prime}\left(c_{t}\right) \sum_{i=t+1}^{+\infty} \frac{s_{i}}{s_{t+1}} u\left(c_{i}\right) \exp \left(-\sum_{j=t}^{i-1} v\left(c_{j}\right)\right)}{u^{\prime}\left(c_{t}\right)-v^{\prime}\left(c_{t}\right) \sum_{i=t+1}^{+\infty} \frac{s_{i}}{s_{t}} u\left(c_{i}\right) \exp \left(-\sum_{j=t}^{i-1} v\left(c_{j}\right)\right)} \\
= & \frac{s_{t+1}}{s_{t}}\left(1-\frac{u^{\prime}\left(c_{t}\right)\left(1-e^{-v\left(c_{t}\right)}\right)-v^{\prime}\left(c_{t}\right) u\left(c_{t}\right) e^{-v\left(c_{t}\right)}}{u^{\prime}-v^{\prime} \sum_{i=t+1}^{+\infty} \frac{s_{i}}{s_{k}} u\left(c_{i}\right) \exp \left(-\sum_{j=t}^{i-1} v\left(c_{j}\right)\right)}\right) \\
& +\frac{s_{t+1}}{s_{t}}\left(\frac{v^{\prime}\left(c_{t}\right) \sum_{i=t+1}^{+\infty}\left(\frac{s_{i}}{s_{t}}-\frac{s_{i}}{s_{t+1}}\right) u\left(c_{i}\right) \exp \left(-\sum_{j=t}^{i-1} v\left(c_{j}\right)\right)}{u^{\prime}\left(c_{t}\right)-v^{\prime}\left(c_{t}\right) \sum_{i=t+1}^{+\infty} \frac{s_{i}}{s_{k}} u\left(c_{i}\right) \exp \left(-\sum_{j=t}^{i-1} v\left(c_{j}\right)\right)}\right)
\end{aligned}
$$

Now remarking that:

$$
\frac{s_{t+1}}{s_{t}}\left(\frac{s_{i}}{s_{t}}-\frac{s_{i}}{s_{t+1}}\right)=\frac{s_{i}}{s_{t}}\left(\frac{s_{t+1}}{s_{t}}-1\right)=-\mu_{t} \frac{s_{i}}{s_{t}}
$$

we find that the term (27) equals:

$$
-\mu_{t} v^{\prime}\left(c_{t}\right) \frac{v^{\prime}\left(c_{t}\right) \sum_{i=t+1}^{+\infty} \frac{s_{i}}{s t} u\left(c_{i}\right) \exp \left(-\sum_{j=t}^{i-1} v\left(c_{j}\right)\right)}{u^{\prime}\left(c_{t}\right)-v^{\prime}\left(c_{t}\right) \sum_{i=t+1}^{+\infty} \frac{s_{i}}{s_{t}} u\left(c_{i}\right) \exp \left(-\sum_{j=t}^{i-1} v\left(c_{j}\right)\right)}
$$

which is nothing else than $-\mu_{t} \rho_{\mu, t} V S L_{\mu, t}$.

Substituting $\frac{s_{t+1}}{s_{t}}$ by $\left(1-\mu_{t}\right)$ in $(26)$, we get:

$$
\begin{aligned}
R T P_{\mu, t}(c)= & 1-\frac{\frac{\partial}{\partial c_{t+1}} E_{\mu, 0} U(c)}{\frac{\partial}{\partial c_{t}} E_{\mu, 0} U(c)} \\
= & \mu_{t}+\left(1-\mu_{t}\right) \frac{u^{\prime}\left(c_{t}\right)\left(1-e^{-v\left(c_{t}\right)}\right)-v^{\prime}\left(c_{t}\right) u\left(c_{t}\right) e^{-v\left(c_{t}\right)}}{u^{\prime}\left(c_{t}\right)-v^{\prime}\left(c_{t}\right) \sum_{i=t+1}^{+\infty} \frac{s_{i}}{s_{t}} u\left(c_{i}\right) \exp \left(-\sum_{j=t}^{i-1} v\left(c_{j}\right)\right)} \\
& +\mu_{t} \rho_{t} V S L_{\mu, t}
\end{aligned}
$$

In a deterministic setting where people would die at the end of period $T>t$, we know from (6) that:

$$
R T P_{t}(c, T)=\frac{u^{\prime}\left(c_{t}\right)\left(1-e^{-v\left(c_{t}\right)}\right)-v^{\prime}\left(c_{t}\right) u\left(c_{t}\right) e^{-v\left(c_{t}\right)}}{u^{\prime}\left(c_{t}\right)-v^{\prime}\left(c_{t}\right) \sum_{i=t+1}^{T} u\left(c_{i}\right) \exp \left(-\sum_{j=t}^{i-1} v\left(c_{j}\right)\right)}
$$


Thus

$$
\frac{u^{\prime}\left(c_{t}\right)\left(1-e^{-v\left(c_{t}\right)}\right)-v^{\prime}\left(c_{t}\right) u\left(c_{t}\right) e^{-v\left(c_{t}\right)}}{u^{\prime}\left(c_{t}\right)-v^{\prime}\left(c_{t}\right) \sum_{i=t+1}^{+\infty} \frac{s_{i}}{s_{t}} u\left(c_{i}\right) \exp \left(-\sum_{j=t}^{i-1} v\left(c_{j}\right)\right)}=\frac{1}{E_{\mu, t}\left(\frac{1}{R T P_{t}(c, .)}\right)}
$$

which leads to

$$
R T P_{\mu, t}(c)=\mu_{t}+\left(1-\mu_{t}\right) \frac{1}{E_{\mu, t}\left(\frac{1}{R T P_{t}(c, .)}\right)}+\mu_{t} \rho_{t} V S L_{\mu, t}
$$

which end proving Proposition 2.

As mentioned in the comments coming after Proposition 2, looking at how mortality at age $t$ impacts the rate of time discounting at time $t$ may be a way to identify time preferences and intertemporal correlation aversion. However, in order to do so, one has to account for the fact that both $E_{\mu, t}\left(\frac{1}{R T P_{t}(c, .)}\right)$ and $V S L_{\mu, t}$ depend on $\mu_{t}$. The object of the lines below, is to explicit this dependence and to explain that this only generates second order-terms, when the length of the time period tends towards zero.

From $(23)$ and $\frac{1}{s_{t}}=\left(1-\mu_{t}\right) \frac{1}{s_{t+1}}$ one can rewrite:

$$
V S L_{\mu, t}=\frac{1}{\frac{1}{\widetilde{V S} L_{\mu, t}}+\mu_{t} v^{\prime}\left(c_{t}\right)}
$$

where $\widetilde{V S L}_{\mu, t}$, defined by,

$$
\widetilde{V S L}_{\mu, t}=\frac{\sum_{i=t+1}^{+\infty} \frac{s_{i}}{s_{t+1}} u\left(c_{i}\right) \exp \left(-\sum_{j=1}^{i-1} v\left(c_{j}\right)\right)}{u^{\prime}\left(c_{t}\right)-v^{\prime}\left(c_{t}\right) \sum_{i=t+1}^{+\infty} \frac{s_{i}}{s_{t+1}} u\left(c_{i}\right) \exp \left(-\sum_{j=1}^{i-1} v\left(c_{j}\right)\right)}
$$

is independent of $\mu_{t}$.

Moreover, using (10), (11), $\frac{s_{t+1}}{s_{t}}=1-\mu_{t}$ and $\mu_{t}=\frac{\pi_{t}}{s_{t}}$ one gets:

$$
E_{\mu, t} f(x, .)=E_{\mu, t+1} f(x, .)+\mu_{t}\left[f(x, t)-E_{\mu, t+1} f(x, .)\right]
$$


Combining (28), (29) and (30) we obtain:

$$
\begin{aligned}
R T P_{\mu, t}(c)= & \mu_{t}+ \\
& \left(1-\mu_{t}\right) \frac{1}{E_{\mu, t+1}\left(\frac{1}{R T P_{t}(c, .)}\right)+\mu_{t}\left[R T P_{t}(c, t)-E_{\mu, t+1}\left(\frac{1}{R T P_{t}(c, .)}\right)\right]} \\
& +\mu_{t} \rho_{t} \frac{1}{\frac{1}{\widetilde{V S} L_{\mu, t}}+\mu_{t} v^{\prime}\left(c_{t}\right)}
\end{aligned}
$$

where all dependence in $\mu_{t}$ is now made explicit (in the above expression, the terms $E_{\mu, t+1}\left(\frac{1}{R T P_{t}(c, .)}\right), R T P_{t}(c, t)$ and $\widetilde{V S L}_{\mu, t}$ are independent from $\left.\mu_{t}\right)$.

The expression shown in (31) seems complex. However, it simplifies when considering the case where the length of the time period is small. Indeed, when the length of the time period tends towards zero, the mortality rate as well as the rate of time discounting decrease proportionally to the length of the time period and many second-order terms, decreasing as the square of the length of the time period, can be neglected. Neglecting all second-order terms, we end up with a very simple formula:

$$
R T P_{\mu, t}(c) \simeq \frac{1}{E_{\mu, t+1}\left(\frac{1}{R T P_{t}(c, .)}\right)}+\mu_{t}\left(1+\rho_{t} \widetilde{V S L}_{\mu, t}\right)
$$

where $E_{\mu, t+1}\left(\frac{1}{R T P_{t}(c, .)}\right)$ and $\rho_{t} \widetilde{V S L}_{\mu, t}$ are independent from $\mu_{t}$.

Since $\left(E_{\mu, t+1}\left(\frac{1}{R T P_{t}(c, .)}\right)-E_{\mu, t}\left(\frac{1}{R T P_{t}(c, .)}\right)\right)$ and $\mu_{t}\left(\widetilde{V S L}_{\mu, t}-V S L_{\mu, t}\right)$ are also second order terms, we may write as well:

$$
R T P_{\mu, t}(c) \simeq \frac{1}{E_{\mu, t}\left(\frac{1}{R T P_{t}(c, .)}\right)}+\mu_{t}\left(1+\rho_{t} V S L_{\mu, t}\right)
$$

This approximation is exact for the additive model, or when assuming infinitesimally small periods of time.

\section{References}

[1] Ahn, C. M., 1989, "The Effect of Temporal Risk Aversion on Optimal Consumption, the Equity Premium, and the Equilibrium Interest Rate". The Journal of Finance, 44(5):1411-20. 
[2] Andersen, S., Harrison, G., Lau, M. and E. Rutström, 2011, Intertemporal Utility and Correlation Aversion. Working paper of the Center for the Economic Analysis of Risk, Georgia State University.

[3] Attanasio,O., Banks, J., Meghir, C. and G. Weber, 1999, "Humps and Bumps in Lifetime Consumption". Journal of Business 83 Economic Statistics, 17(1):22-35

[4] Becker, G. S., Philipson, T. J. and R. R. Soares, 2005, "The Quantity and Quality of Life and the Evolution of World Inequality". The American Economic Review, 95(1):277-291.

[5] Bloom, D. E., Canning, D. and B. Graham, 2003, "Longevity and Life-cycle Savings". The Scandinavian Journal of Economics, 105(3): 319 -338.

[6] Bommier, A., 2006, "Uncertain Lifetime and Intertemporal Choice: Risk Aversion as a Rationale for Time Discounting". International Economic Review, 47(4):1223-1246.

[7] Bommier, A., 2007, "Risk Aversion, Intertemporal Elasticity of Substitution and Correlation Aversion". Economics Bulletin, 4(29):1-8.

[8] Bommier, A., 2008, Rational Impatience?, mimeo, University of Toulouse.

[9] Bommier, A., 2010, "Portfolio choice under uncertain lifetime". Journal of Public Economic Theory, 12(1) 57-73.

[10] Bommier, A., Chassagnon, A. and F. Le Grand, 2010, "Comparative Risk Aversion: A formal Approach with Applications to Saving Behaviors". Journal of Economic Theory, forthcoming.

[11] Bommier, A., Leroux M.-L. and J.-M. Lozachmeur, 2010, "On the Public Economics of Annuities with Differential Mortality". Journal of Public Economics, forthcoming.

[12] Bommier, A. and J.-C. Rochet, 2006, "Risk Aversion and Planning Horizons". Journal of the European Economic Association, 4(4):708-734.

[13] Bommier, A. and B. Villeneuve, 2010, "Risk Aversion and the Value of Risk to Life". Journal of Risk and Insurance, forthcoming.

[14] Boucekkine R., de la Croix, D. and O. Licandro, 2003, "Early Mortality Declines at the Dawn of Modern Growth". Scandinavian Journal of Economics, 105(3):401-418

[15] Bütler, M. , 2001, "Neoclassical life-cycle consumption: a textbook example". Economic Theory 17(1): 209-221.

[16] Cavalcanti Ferreira, P. and S. Pessôa, 2003, The Long Run Economic Impact of AIDS. Ensaios Econômicos EPGE no. 475. 
[17] Diamond, P.A., 1965, "The Evaluation of Infinite Utility Streams"” , Econometrica, 33(1):170-177.

[18] Dorfleitner, G. and M. Krapp, 2007, "On multiattributive risk aversion: some clarifying results". Review of Managerial Science, 1(1):47-63.

[19] Eeckhoudt, L., Rey, B. and H. Schlesinger, (2007). "A Good Sign for Multivariate Risk Taking," Management Science, 53(1): 117-124.

[20] Epstein, L. G, 1983, "Stationary Cardinal Utility and Optimal Growth Under Uncertainty". Journal of Economic Theory, 31: 133-52.

[21] Epstein, L. G. and S. M. Tanny, 1980, "Increasing Generalized Correlation: A Definition and Some Economic Consequences". The Canadian Journal of Economics, 13(1):16-34.

[22] Epstein, L. G. and S. E. Zin, 1989, "Substitution, Risk Aversion, and the Temporal Behavior of Consumption and Asset Returns: A Theoretical Framework". Econometrica, 57(4): 937-969.

[23] Feigenbaum, J., 2008, "Can Mortality Risk Explain the Consumption Hump?". Journal of Macroeconomics 30: 844-872.

[24] Fernández-Villaverde, J. and D. Krueger, 2007, "Consumption over the Life Cycle: Facts from Consumer Expenditure Survey Data". The Review of Economics and Statistics 89(3):552-565.

[25] de Finetti, B., 1952, "Sulla preferibilità". Giornale degli Economisti e Annali di Economia, 11:685-709.

[26] Fishburn, P. C., 1970, Utility Theory for Decision-Making. John Wiley and Sons, New-York.

[27] Gourinchas, P.-O. and J. Parker, 2002, "Consumption Over the Life Cycle". Econometrica 70(1): 47-89.

[28] Hansen, G. and S. Imrohoroglu, 2008, "Consumption over the Life Cycle: The Role of Annuities," Review of Economic Dynamics 11(3): 566-583,

[29] Johansson, P.-O., 2002, "On the Definition and Age-Dependency of the Value of a Statistical Life". Journal of Risk and Uncertainty, 25(3): 251-263.

[30] Keeney, R. L. and H. Raiffa, (eds), 1976, Decisions with Multiple Objectives. John Wiley and Sons (re-edited in 1993 by Cambridge University Press).

[31] Kihlstrom, R.E. and L.J. Mirman, 1974, "Risk Aversion with many Commodities". Journal of Economic Theory 8:361-88.

[32] Koopmans, T.C., 1960, "Stationary Ordinal Utility and Impatience". Econometrica, 28(2):287-309. 
[33] Kreps, D. M. and E. L. Porteus, 1978, "Temporal Resolution of Uncertainty and Dynamic Choice Theory". Econometrica, 46(1): 185-200.

[34] Mas-Colell, A., M. Whinston, and J. Green, 1995, Microeconomic Theory. Oxford University Press.

[35] Peart, S. J. and D. M. Levy, 2003, "Denying Human Homogeneity: Eugenics \& the Making of Post-Classical Economics". Journal of the History of Economic Thought 25(3): 261 - 288.

[36] Pye, G., 1973, "Lifetime Portfolio Selection in Continuous Time for a Multiplicative Class of Utility Functions". The American Economic Review, 63(5): 1013-1016.

[37] Ramsey, F., 1928, A Mathematical Theory of Saving, The Economic Journal, 38(152):543-559.

[38] Richard, S. F., 1975, "Multivariate Risk Aversion, Utility Independence and Separable Utility Functions". Management Science, 22(1): 12-21.

[39] Tsetlin, I and R. Winkler, 2009, " Multiattribute Utility Satisfying a Preference for Combining Good with Bad". Management Science, 55(12):1942-52.

[40] Uzawa, H, 1968, "Time preference, the consumption function, and optimum asset holdings". In Value, Capital, and Growth: Papers in Honor of Sir John Hicks (J. N. Wolfe, Ed.), Edinburgh University, Edinburgh, 1968.

[41] Van der Ploeg, F., 1993, "A Closed-form Solution for a Model of Precautionay Saving". The Review of Economic Studies, 60(2):385-395.

[42] Viscusi, W. K. and J. E. Aldy, 2003, "The Value of a Statistical Life: A Critical Review of Market Estimates throughout the World". Journal of Risk and Uncertainty 27(1):5-76.

[43] Vissing-Jørgensen, A. and 0. Attanasio, 2003, "Stock-Market Participation, Intertemporal Substitution, and Risk-Aversion". The American Economic Review, 93(2):383-391.

[44] Wakker, P. P., S. Jansen and A. M. Stiggelbout, 2004, "Anchor Levels as a New Tool for the Theory and Measurement of Multiattribute Utility". Decision Analysis 1:217-234.

[45] Yaari, M. E., 1965, "Uncertain Lifetime, Life Insurance, and the Theory of the Consumer". The Review of Economic Studies, 32(2): 137-150. 
Figure 1: The set of stationary preferences

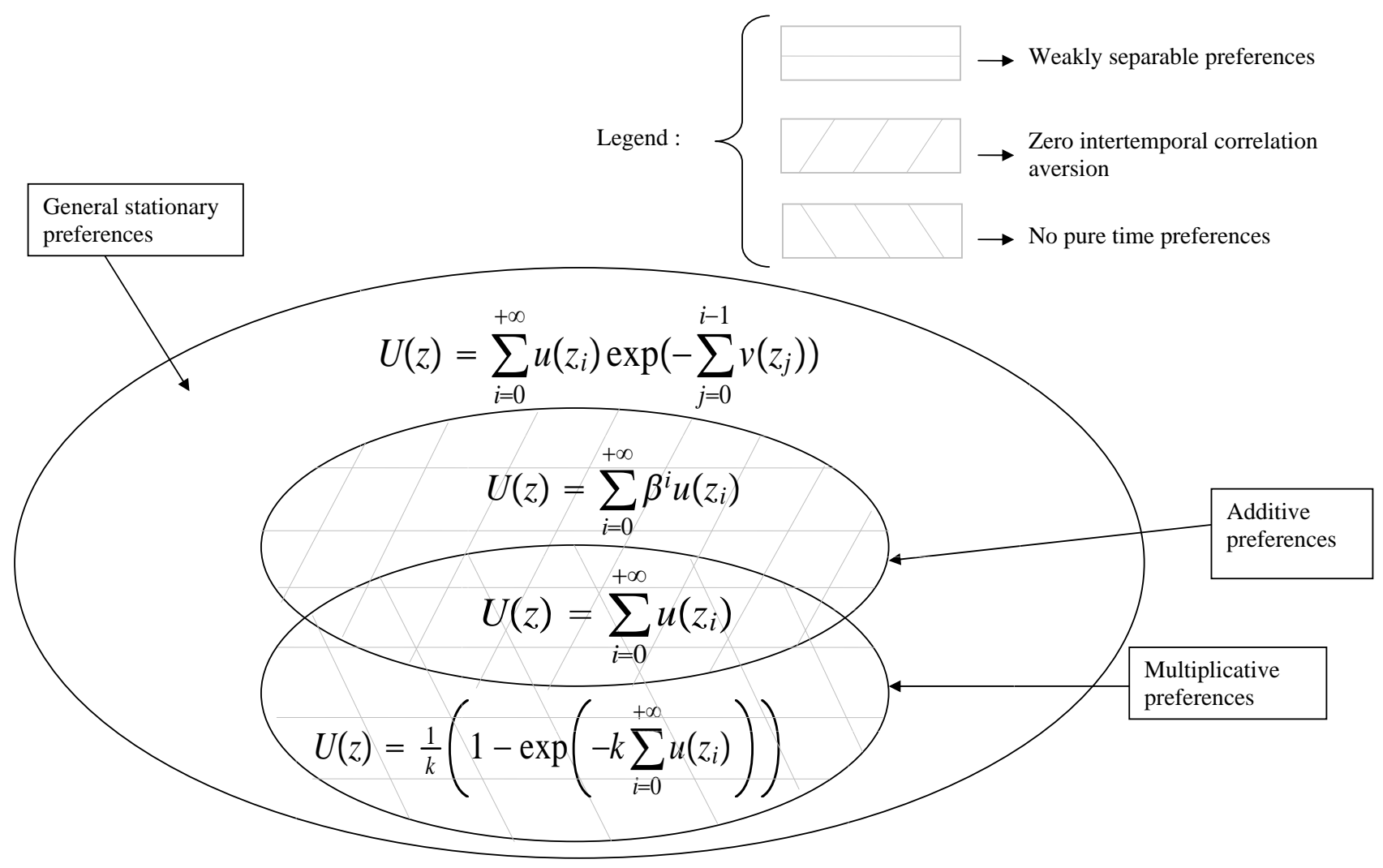


Figure 2: Life-Cycle Consumption Profile (Best Fit)

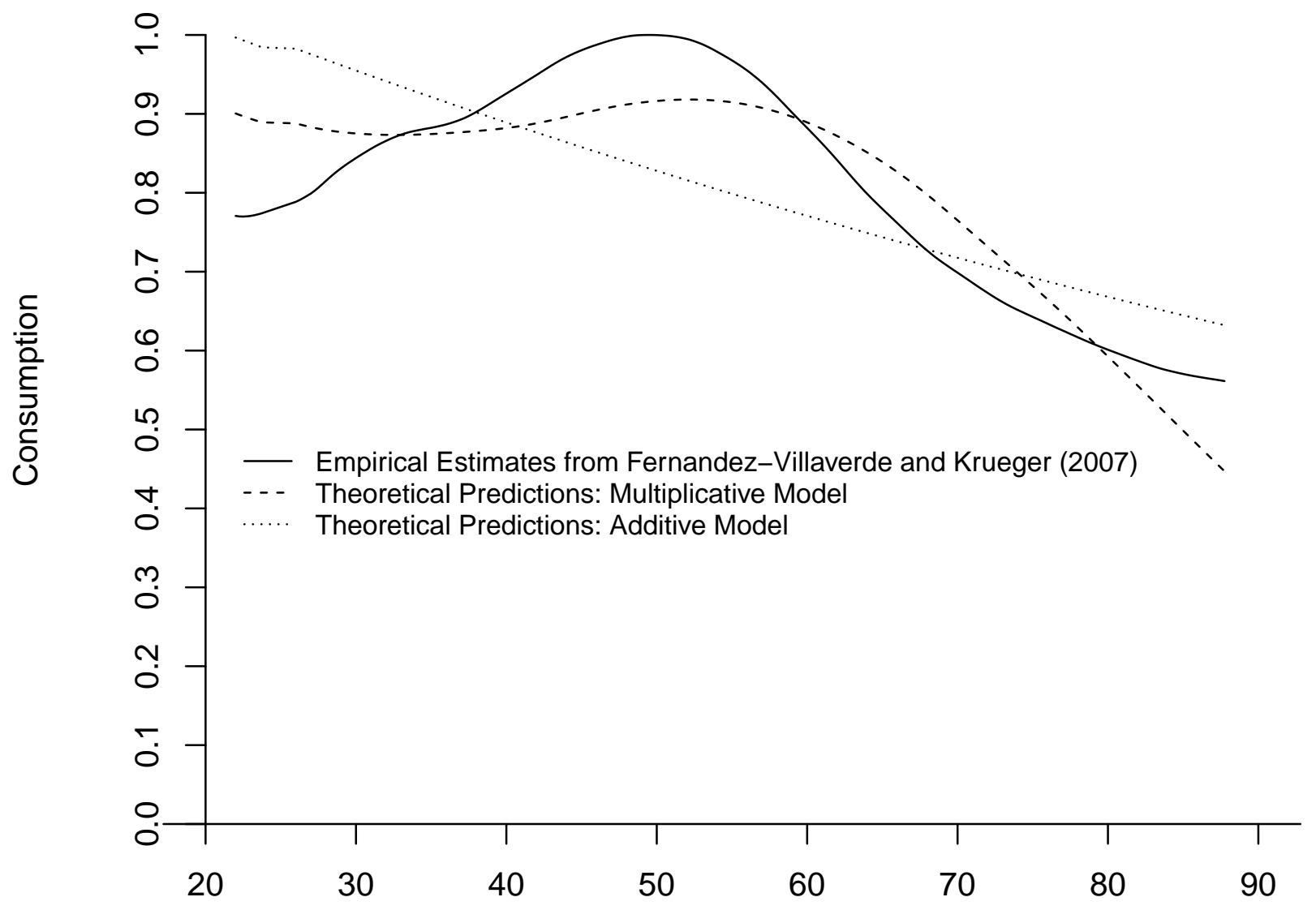

Age (years) 
Figure 3: Life-Cycle Consumption Profile (imposing IES=1)

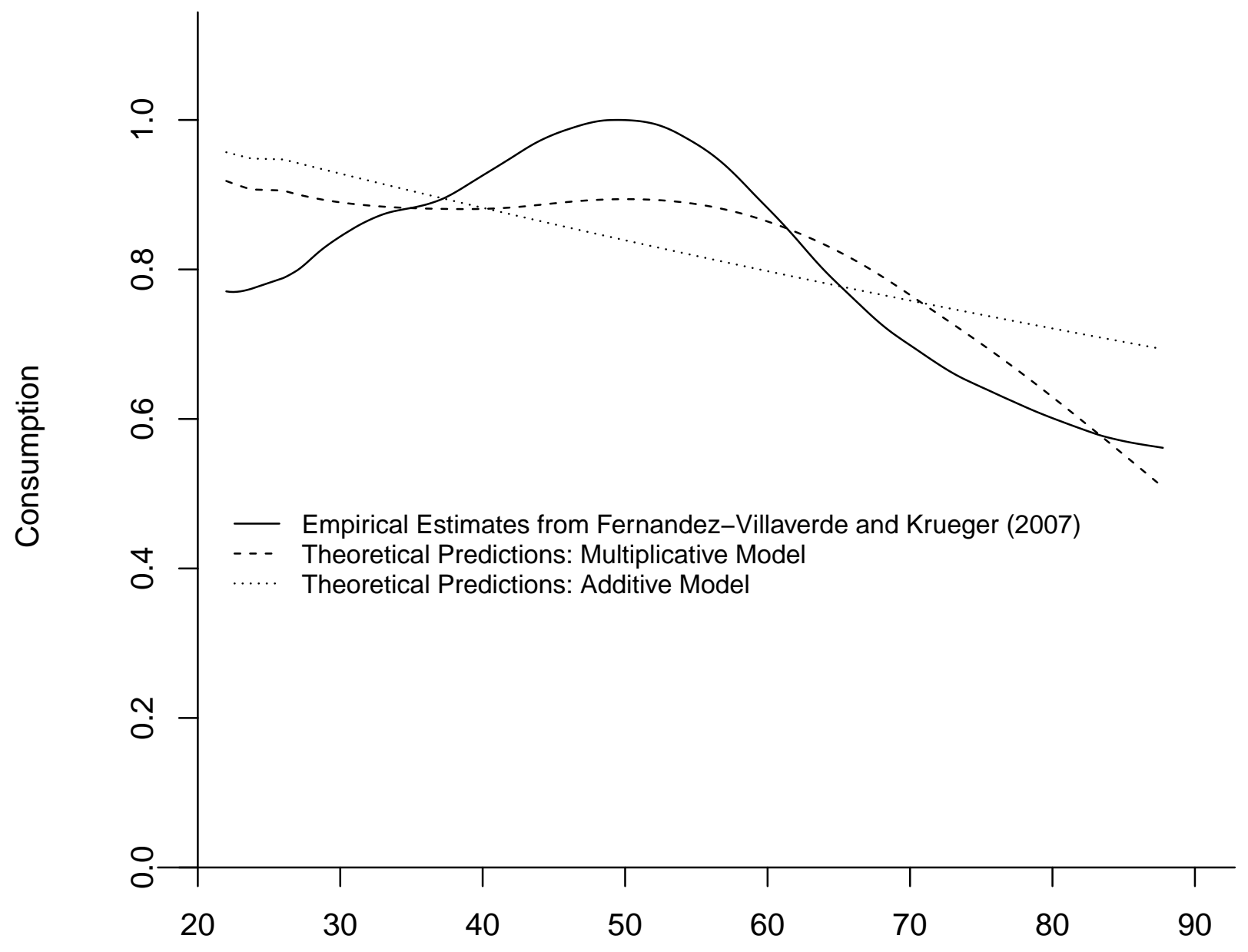

Age (years) 


\section{Working Papers of the Center of Economic Research at ETH Zurich}

(PDF-files of the Working Papers can be downloaded at www.cer.ethz.ch/research).

11/154 C. N. Brunnschweiler and S. Valente

International Partnerships, Foreign Control and Income Levels: Theory and Evidence

11/153 R. Ramer

Dynamic Effects and Structural Change under Environmental Regulation in a CGE Model with Endogenous Growth

11/152 I. A. MacKenzie and M. Ohndorf Optimal Monitoring of Credit-based Emissions Trading under Asymmetric Information

11/151 J. Daubanes and P. Lasserre Optimum Commodity Taxation with a Non-Renewable Resource

11/150 A. Schäfer and M. T. Schneider Endogenous Enforcement of Intellectual Property, North-South Trade, and Growth

11/149 H. Gersbach and V. Hahn Inflation Forecast Contracts

11/148 D. Schiess and R. Wehrli Long-Term Growth Driven by a Sequence of General Purpose Technologies

11/147 P. F. Peretto and S. Valente Growth on a Finite Planet: Resources, Technology and Population in the Long Run

11/146 H. Gersbach, N. Hummel and R. Winkler Sustainable Climate Treaties

11/145 H. Gersbach and H. Haller A Human Relations Paradox

11/144 L. Bretschger and S. Valente International Trade and Net Investment: Theory and Evidence

11/143 H. Gersbach Campaigns, Political Mobility, and Communication

11/142 J. G. Becker On the Number of $\alpha$-Pivotal Players

11/141 P. S. Schmidt, U. von Arx, A. Schrimpf, A. F. Wagner and A. Ziegler On the Construction of Common Size, Value and Momentum Factors in International Stock Markets: A Guide with Applications 
10/140 L. Leinert

How do unanticipated discoveries of oil fields affect the oil price?

10/139 H. Gersbach, M. T. Schneider and O. Schneller

Basic Research, Openness, and Convergence

10/138 L. Bretschger and V. Kappel

Market concentration and the likelihood of financial crises

10/137 M. T. Schneider and R. Winkler

Growth and Welfare under Endogenous Lifetime

10/136 V. Hahn

Sequential Aggregation of Verifiable Information

10/135 A. Bommier, M.-L. Leroux and J.-M. Lozachmeur

On the Public Economics of Annuities with Differential Mortality

10/134 A. Bommier, A. Chassagnon and F. Le Grand

Comparative Risk Aversion: A Formal Approach with Applications to Saving Behaviors

10/133 A. Bommier and B. Villeneuve

Risk Aversion and the Value of Risk to Life

10/132 L. Bretschger and S. Valente

Endogenous Growth, Asymmetric Trade and Resource Taxation

10/131 H. Gersbach and N. Surulescu

Default Risk in Stochastic Volatility Models

10/130 F. Schwark

Economics of Endogenous Technical Change in CGE Models - The Role of Gains from Specialization

10/129 L. Bretschger, R. Ramer and F. Schwark

Long-Run Effects of Post-Kyoto Policies: Applying a Fully Dynamic CGE model with Heterogeneous Capital

10/128 M. T. Schneider, C. Traeger and R. Winkler

Trading Off Generations: Infinitely-Lived Agent Versus OLG

10/127 V. Kappel

The Effects of Financial Development on Income Inequality and Poverty

10/126 M. T. Schneider

The Larger the Better? The Role of Interest-Group Size in Legislative Lobbying 
10/125 A. Ziegler

Individual Characteristics and Stated Preferences for Alternative Energy Sources and Propulsion Technologies in Vehicles: A Discrete Choice Analysis

10/124 P. F. Peretto and S. Valente

Resource Wealth, Innovation and Growth in the Global Economy

09/123 H. Gersbach and M. T. Schneider

Tax Contracts and Elections

09/122 V. Hahn

Why the Publication of Socially Harmful Information May Be Socially Desirable

09/121 A. Ziegler

Is it Beneficial to be Included in a Sustainability Stock Index? A Panel Data Study for European Firms

09/120 K. Pittel and L. Bretschger

The Implications of Heterogeneous Resource Intensities on Technical Change and Growth

09/119 E. J. Balistreri, R. H. Hillberry and T. F. Rutherford

Trade and Welfare: Does Industrial Organization Matter?

09/118 H. Gersbach, G. Sorger and C. Amon

Hierarchical Growth: Basic and Applied Research

09/117 C. N. Brunnschweiler

Finance for Renewable Energy: An Empirical Analysis of Developing and Transition Economies

09/116 S. Valente

Optimal Policy and Non-Scale Growth with R\&D Externalities

09/115 T. Fahrenberger

Short-term Deviations from Simple Majority Voting

09/114 M. Müller

Vote-Share Contracts and Learning-by-Doing

09/113 C. Palmer, M. Ohndorf and I. A. MacKenzie

Life's a Breach! Ensuring 'Permanence' in Forest Carbon Sinks under Incomplete Contract Enforcement

09/112 N. Hanley and I. A. MacKenzie

The Effects of Rent Seeking over Tradable Pollution Permits 